Max Reinwald/Hendrik Hüttermann/Julia Kröll/Sabine Boerner*

\title{
Gender Diversity in Führungsteams und Unternehmensperformanz: Eine Metaanalyse**
}

\section{Zusammenfassung}

Theoretische und empirische Untersuchungen des Zusammenhangs zwischen Gender Diversity in Führungsteams und Unternehmensperformanz weisen inkonsistente Befunde auf. Vor diesem Hintergrund präsentiert der vorliegende Beitrag die Ergebnisse einer Metaanalyse zu der Frage, wie und in Abhängigkeit von welchen Randbedingungen eine gemischt-geschlechtliche Zusammensetzung von Führungsteams mit Unternehmensperformanz verbunden ist. Basierend auf 38 Einzelstudien kann kein signifikanter Zusammenhang zwischen Gender Diversity und Unternehmensperformanz insgesamt identifiziert werden. Zudem erweisen sich auch die untersuchten Moderatoren (Größe des Führungsteams, Branche, kultureller Kontext und Performanzkriterium) als nicht signifikant.

JEL-Classification: $\quad$ M12, M50, M54.

Keywords: Führungsteams; Gender Diversity; Metaanalyse; Moderatoren; Unternehmensperformanz.

Gender Diversity; Leadership Teams; Meta-Analysis; Moderators; Organizational Performance.

* Max Reinwald, B.A., Studierender im Master-Studiengang Politik- und Verwaltungswissenschaft an der Universität Konstanz, Postanschrift: Universitätsstraße 10, 78464 Konstanz, E-Mail: max.reinwald@uni-konstanz.de; Dr. Hendrik Hüttermann, Wissenschaftlicher Projektleiter und Lehrbeauftragter an der Universität St.Gallen, Institut für Führung und Personalmanagement, Postanschrift: Dufourstrasse 40a, 9000 St.Gallen, Schweiz, EMail: hendrik.huettermann@unisg.ch; Julia Kröll, Dipl.-Psych., Wissenschaftliche Mitarbeiterin an der Universität Konstanz, Lehrstuhl für Management, insbesondere Strategie und Führung, Postanschrift: Universitätsstraße 10, 78464 Konstanz, E-Mail: julia.kroell@uni-konstanz.de; Prof. Dr. Sabine Boerner, Professorin an der Universität Konstanz, Lehrstuhl für Management, insbesondere Strategie und Führung, Postanschrift: Universitätsstraße 10, 78464 Konstanz, E-Mail: sabine.boerner@uni-konstanz.de.

** Die Autoren danken Tabea Friedemann für ihre Unterstützung bei der Literaturrecherche sowie einem anonymen Gutachter für wertvolle Hinweise und Anmerkungen. 


\section{Einleitung}

Die Beschäftigtenstruktur in Deutschland wird immer vielfältiger. So haben gesellschaftliche Veränderungsprozesse wie der demographische Wandel und die wachsende internationale Mobilität von Arbeitskräften dazu geführt, dass die Belegschaften deutscher Unternehmen eine zunehmende „Diversity“ aufweisen ${ }^{1}$. Diversity bezeichnet in diesem Zusammenhang Unterschiede zwischen Mitarbeitern in Bezug auf ein bestimmtes Merk$\mathrm{mal}^{2}$. Ein Diversity-Merkmal, das in der aktuellen öffentlichen Diskussion besondere Beachtung findet, ist das Geschlecht der Mitarbeiter (sogenannte Gender Diversity) ${ }^{3,4}$. Ausschlaggebend für die gegenwärtige Relevanz von Gender Diversity sind dabei unter anderem gesellschaftliche Entwicklungen wie die zunehmende Zahl gut qualifizierter Arbeitnehmerinnen und die wachsende Frauenerwerbstätigkeit ${ }^{5}$.

Trotz des steigenden Frauenanteils in den Gesamtbelegschaften weisen deutsche Unternehmen jedoch beim Frauenanteil in den obersten Führungsgremien noch Nachholbedarf auf ${ }^{6}$. So waren im Jahr 2014 lediglich 7,4\% der Vorstandsmitglieder und 24,7\% der Aufsichtsratsmitglieder in den DAX-30-Unternehmen weiblich ${ }^{7}$, verglichen mit einem Frauenanteil an der Gesamt-Erwerbsbevölkerung von $46,1 \%{ }^{8}$. Nicht zuletzt vor dem Hintergrund dieser Diskrepanz wird der gesellschaftliche Diskurs in den vergangenen Jahren maßgeblich durch die Frage geprägt, wie eine Erhöhung des Frauenanteils in Führungspositionen erreicht werden kann. In diesem Zusammenhang wurden in Deutschland verschiedene politische Initiativen auf den Weg gebracht, wie etwa eine gesetzlich festgeschriebene Selbstverpflichtung von börsennotierten Unternehmen in Form einer so genannten „Flexi-Quote“9. Außerdem hat der Bundestag Anfang 2015 ein Gesetz verabschiedet, das ab 2016 eine Frauenquote von 30\% für die Aufsichtsräte von rund 100 großen börsennotierten Unternehmen vorschreibt ${ }^{10}$.

Dabei stellt sich aus Sicht der Unternehmen - unabhängig von der gesellschaftlich-normativen Legitimation einer Frauenförderung (so genannter „moral justice case “11) - die Frage, welche Auswirkungen eine erhöhte Gender Diversity in Führungsgremien (das heißt in Aufsichtsräten und Vorständen) für die jeweilige Organisation hat. In diesem $\mathrm{Zu}$ sammenhang dürften insbesondere leistungsrelevante Auswirkungen von Gender Diver-

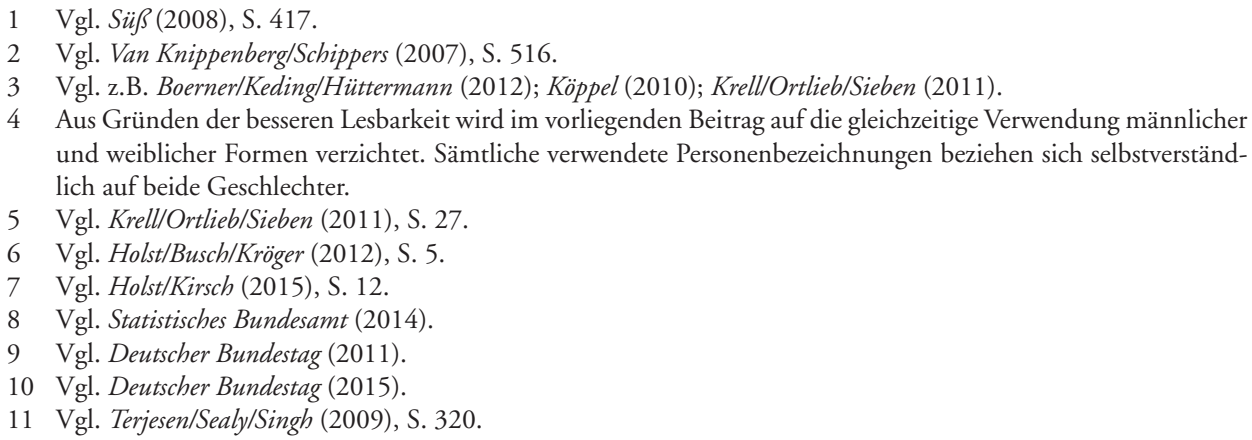
und weiblicher Formen verzichtet. Sämtliche verwendete Personenbezeichnungen beziehen sich selbstverständlich auf beide Geschlechter. 
sity in Führungsgremien von Bedeutung sein (sogenannter „business case“12). Obgleich die Verbindung zwischen Gender Diversity in Führungsgremien und Unternehmensperformanz bislang kaum im deutschen Kontext untersucht wurde ${ }^{13}$, existiert eine Reihe internationaler Studien zu diesem Zusammenhang. Diese weisen jedoch widersprüchliche Befunde auf und zeigen sowohl positive als auch negative und nicht signifikante Effekte ${ }^{14}$. Eine systematische Aufarbeitung des Forschungsstandes wurde aber bislang nicht vorgenommen; so untersuchen bestehende Metaanalysen weder explizit Gender Diversity in Führungsteams noch Performanzeffekte auf Unternehmensebene ${ }^{15}$.

Vor diesem Hintergrund ist es das Ziel des vorliegenden Beitrags, den Zusammenhang zwischen Gender Diversity in Führungsgremien und Unternehmensperformanz erstmals im Rahmen einer Metaanalyse zu untersuchen. Neben der direkten Verbindung zwischen den beiden Variablen werden dabei auch Randbedingungen (sogenannte „Moderatoren “16) des Zusammenhangs analysiert. Eine Untersuchung von Moderatoren kann möglicherweise Aufschluss darüber geben, unter welchen Randbedingungen Gender Diversity in Führungsgremien positiv, negativ oder nicht signifikant mit Unternehmensperformanz verbunden ist.

\section{Theorie und Hypothesen}

\subsection{Gender Diversity in Führungsteams und Unternehmensperformanz}

Mit Blick auf die Effekte einer gemischt-geschlechtlichen Zusammensetzung von Führungsteams existieren in der Diversity-Forschung zwei unterschiedliche theoretische Perspektiven. Diese lassen widersprüchliche Vorhersagen hinsichtlich der Auswirkungen auf Unternehmensperformanz $\mathrm{zu}^{17}$.

Einerseits kann im Sinne der Informations- und Entscheidungsperspektive ein positiver Effekt von Gender Diversity auf die Unternehmensleistung erwartet werden. Dabei wird angenommen, dass weibliche und männliche Mitglieder unterschiedliche aufgabenbezogene Wissens- und Erfahrungshintergründe, Sichtweisen und persönliche Stärken in ein Führungsteam einbringen können ${ }^{18}$. In diesem Zusammenhang führen beispielsweise verschiedene Autoren an, dass Frauen verbal versierter, prozessorientierter und weniger riskant agieren als ihre männlichen Kollegen. Männern hingegen werden insbesondere in Bezug auf Durchsetzungskraft, Aufgabenorientierung und räumliches Vorstellungs-

12 Vgl. Krell/Ortlieb/Sieben (2011), S. 160.

13 Vgl. z.B. Lindstädt/WolffFehre (2011).

14 Vgl. z.B. Lückerath-Rovers (2013); Darmadi (2011); Miller/Triana (2009).

15 Vgl. z.B. Bell et al. (2011); Horwitz/Horwitz (2007); Van Dijk/Van Engen/Van Knippenberg (2012).

16 Ein Moderator ist eine Variable, die einen Einfluss auf die Stärke und/oder Richtung des Zusammenhangs zwischen einer unabhängigen und einer abhängigen Variable besitzt (vgl. Baron/Kenny (1986), S. 1176).

17 Vgl. Van Knippenberg/Schippers (2007), S. 517f.

18 Vgl. Miller/Triana (2009), S. 759 f.; Wegge et al. (2008), S.1303. 
vermögen spezifische Stärken zugeschrieben ${ }^{19}$. Entsprechend verfügen geschlechterheterogene Führungsteams über eine größere Vielfalt an Informationen, Perspektiven und Kompetenzen. Dies kann sich insbesondere bei der Bewältigung wechselnder Herausforderungen in komplexen Unternehmensumwelten als vorteilhaft erweisen ${ }^{20}$. So können die mit Gender Diversity verbundenen differierenden Informationshintergründe dafür sorgen, dass die Teammitglieder bei der Entscheidungsfindung unterschiedliche Blickwinkel berücksichtigen ${ }^{21}$. Dadurch kann die Entscheidungsqualität in gemischtgeschlechtlichen Führungsteams positiv beeinflusst werden ${ }^{22}$.

Andererseits stehen bei der sozialen Kategorisierungsperspektive negative Effekte von Gender Diversity in Führungsteams im Fokus; diese können hauptsächlich durch die Theorie der sozialen Kategorisierung ${ }^{23}$ und die Theorie der sozialen Identität ${ }^{24}$ erklärt werden. Grundgedanke ist, dass es auf Basis wahrgenommener Unterschiede und Gemeinsamkeiten zwischen Teammitgliedern zu sozialen Kategorisierungsprozessen innerhalb von Führungsteams kommt ${ }^{25}$. Steht dabei das Geschlecht der Teammitglieder im Fokus des Kategorisierungsprozesses (d.h. es ist das „saliente“ Diversity-Merkmal26), kann es zur Entstehung von geschlechterspezifischen Subgruppen (männliche versus weibliche Teammitglieder) innerhalb des Teams kommen. Hierbei werden Mitglieder der eigenen Subgruppe in der Regel als vertrauenswürdiger und verlässlicher beurteilt als Mitglieder der anderen Subgruppe ${ }^{27}$. Dies hat zur Folge, dass bevorzugt mit den Mitgliedern der eigenen (männlichen oder weiblichen) Subgruppe kommuniziert und kooperiert wird, was die Zusammenarbeit zwischen den Geschlechtern in Führungsteams beeinträchtigt ${ }^{28}$. Diese Kooperationsdefizite können sich wiederum negativ auf die Performanz der Führungsteams auswirken.

Unter Bezugnahme auf die Upper Echelons-Theorie kann davon ausgegangen werden, dass die beschriebenen positiven und negativen Effekte von Gender Diversity in Führungsteams auch die Performanz der jeweiligen Organisation beeinflussen ${ }^{29}$. So können sich persönliche Unterschiede zwischen den Teammitgliedern (wie z.B. das Geschlecht und damit einhergehende Erfahrungen, Werte und Sichtweisen) auf die strategischen Entscheidungen von Führungsteams auswirken ${ }^{30}$; diese strategischen Entscheidungen wiederum können einen wesentlichen Einfluss auf die Performanz von Unternehmen besitzen.

19 Vgl. Feingold (1994); Halpern (2004); Hirschfeld et al. (2005); Wood (1987).

20 Vgl. Terjesen/Sealy/Singh (2009), S. 329.

21 Vgl. Boerner/Linkohr/Kiefer (2011), S. 331; Jackson/Joshi (2011), S. 658 \& 662.

22 Vgl. Hinsz/Tindale/Vollrath (1997).

23 Vgl. Tajfel (1981); Turner et al. (1987).

24 Vgl. Tajfel/Turner (1986).

25 Vgl. Fiske/Lin/Neuberg (1999), S. 234; Harrison/Price/Bell (1998), S. 98.

26 Vgl. Ashforth/Johnson (2001), S. 32.

27 Vgl. Tajfel/Turner (1986), S. 16; Turner et al. (1987), S. $27 \mathrm{f}$.

28 Vgl. Jehn/Northcraft/Neale (1999), S. 745; Tajfel/Turner (1986), S. 13.

$29 \mathrm{Vgl}$. Hambrick/Mason (1984).

30 Vgl. Bantel/Jackson (1989), S. 110. 
Zusammenfassend lassen die beiden dominierenden theoretischen Perspektiven in der Forschung zu Gender Diversity widersprüchliche Vorhersagen in Bezug auf Unternehmensperformanz zu. Entsprechend werden in einschlägigen integrativen theoretischen Modellen sowohl Informations- und Entscheidungsprozesse als auch Prozesse der sozialen Kategorisierung berücksichtigt ${ }^{31}$. Inwiefern letztlich positive oder negative Effekte überwiegen oder sich gar gegenseitig aufheben, könnte dabei maßgeblich von weiteren Randbedingungen abhängig sein ${ }^{32}$.

Ebenso inkonsistent wie die theoretischen Vorhersagen gestaltet sich auch die empirische Befundlage zum Zusammenhang zwischen Gender Diversity in Führungsteams und Unternehmensperformanz. So identifizieren bestehende Studien positive, negative und nicht signifikante Effekte ${ }^{33}$. Eine systematische metaanalytische Aufarbeitung der empirischen Befunde wurde jedoch bislang nicht vorgenommen. Entsprechend orientiert sich die vorliegende Metaanalyse an folgender Leitfrage: Wie und in Abhängigkeit von welchen Randbedingungen sind Gender Diversity in Führungsteams und Unternehmensperformanz verbunden?

\subsection{Moderatoren des Zusammenhangs zwischen Gender Diversity in Führungsteams und Unternehmensperformanz}

Im Folgenden werden vier Randbedingungen analysiert, die einen Einfluss auf die Richtung und die Stärke des Zusammenhangs zwischen Gender Diversity in Führungsteams und Unternehmensperformanz besitzen können. Diese theoretischen Moderatoren sind gemeinsam mit den ebenfalls untersuchten methodischen Moderatoren (methodische Charakteristika der Einzelstudien; vgl. Kapitel 3) in Abbildung 1 zusammengefasst.

\section{Abbildung 1: Das Moderationsmodell im Überblick}

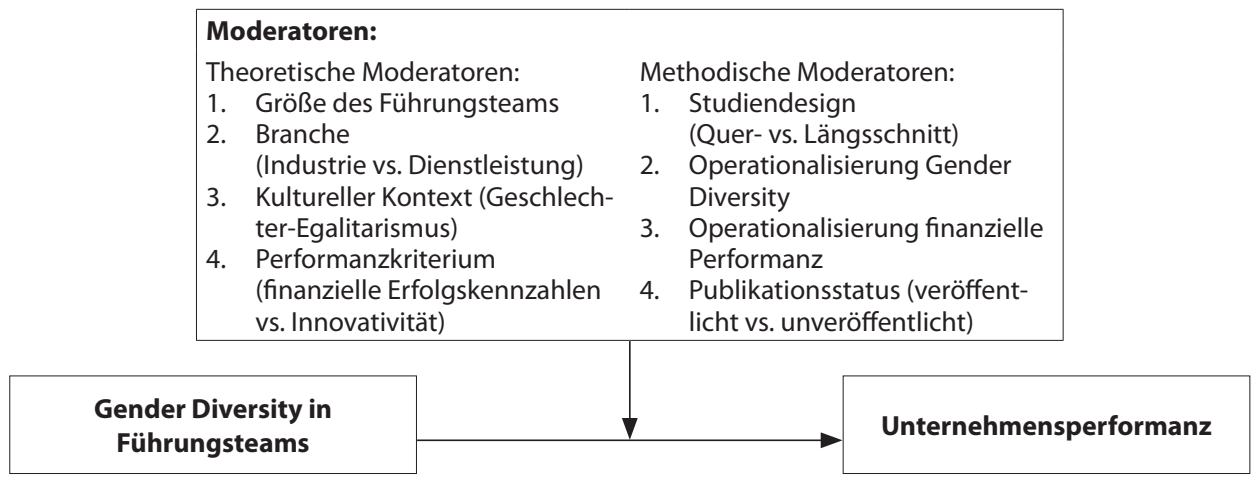

31 Vgl. z.B. Van Knippenberg/De Dreu/Homan (2004).

32 Vgl. Boerner/Keding/Hüttermann (2012); Jackson/Joshi (2011); Van Knippenberg/De Dreu/Homan (2004).

33 Vgl. z.B. Lückerath-Rovers (2013); Darmadi (2011); Miller/Triana (2009). 


\subsubsection{Größe des Führungsteams}

Die Teamgröße kann einen entscheidenden Einfluss auf die in Kapitel 2.1 beschriebenen Informations- und Entscheidungs- sowie sozialen Kategorisierungsprozesse in gemischtgeschlechtlichen Führungsteams besitzen.

Erstens kann davon ausgegangen werden, dass bei zunehmender Größe eines heterogen zusammengesetzten Führungsteams auch dessen Ressourcen und Ressourcenvielfalt zunehmen ${ }^{34}$. So können sich auch Teammitglieder desselben Geschlechts hinsichtlich ihrer geschlechterspezifischen Ressourcen unterscheiden ${ }^{35}$. Entsprechend sollte potentiell jedes zusätzliche Teammitglied weitere geschlechterspezifische Ressourcen in das Team einbringen können und dadurch den informationellen Ressourcenpool vergrößern. Dieser Ressourcenpool kann allerdings die Performanz des Führungsteams nur dann positiv beeinflussen, wenn die Teammitglieder die unterschiedlichen Sichtweisen und Informationen auch tatsächlich nutzen, das heißt austauschen, diskutieren und integrieren ${ }^{36}$. Dies wird jedoch bei zunehmender Teamgröße unwahrscheinlicher. Klein et al. (2009) postulieren, dass in großen Teams verstärkt Informationen geteilt werden, die ohnehin allen Teammitgliedern bekannt sind ${ }^{37}$. Darüber hinaus steigen mit der Zahl der Teammitglieder auch die Komplexität der Interaktion und der Koordinationsaufwand bei der Integration der verschiedenen Sichtweisen ${ }^{38}$. Dadurch kann es zu Prozess- und Kommunikationsverlusten kommen ${ }^{39}$. Es ist zu erwarten, dass dieses Problem auch in Führungsteams besteht, da deren Interaktionsmöglichkeiten in der Regel knapp bemessen sind und sie zudem eine umfassende Agenda besitzen ${ }^{40}$. Dementsprechend bleibt wenig Raum für zeitintensive Koordinationsanstrengungen.

Zweitens steigt mit zunehmender Teamgröße die Gefahr der durch Gender Diversity induzierten sozialen Kategorisierungsprozesse in Führungsteams. So ergibt sich bei zunehmender Geschlechterheterogenität und gleichzeitig wachsender Teamgröße eher die Möglichkeit einer überwiegenden Interaktion mit Personen desselben Geschlechts ${ }^{41}$. Zudem wird mit steigender Teamgröße der Aufbau und Erhalt interpersoneller Beziehungen mit allen Teammitgliedern erschwert ${ }^{42}$. Die Teammitglieder müssen folglich ihre Interaktion auf einige Personen begrenzen ${ }^{43}$, wobei im Sinne des Similarity/Attraction-Paradigmas eine Fokussierung auf Mitglieder des jeweils gleichen Geschlechts erwartet werden $k_{a n n}{ }^{44}$. Dies kann die Bildung von geschlechterspezifischen Subgruppen begünstigen,

34 Vgl. Amason/Sapienza (1997), S. 500; Horwitz/Horwitz (2007), S. 997.

35 Vgl. z.B. Byrnes/Miller/Schafer (1999), S. 377.

36 Vgl. Amason (1996), S. 124; Van Knippenberg/De Dreu/Homan (2004), S. 1011.

37 Vgl. Klein et al. (2009), S. 196.

38 Vgl. Curral et al. (2001), S. 192.

39 Vgl. Gooding/Wagner (1985), S. 476; Guzzo/Shea (1992), S. 281.

40 Vgl. Finkelstein/Mooney (2003), S. 104; Forbes/Milliken (1999), S. 492.

41 Vgl. Wegge et al. (2008), S. 1304.

42 Vgl. Forbes/Milliken (1999), S. 499.

43 Vgl. Turner et al. (1987), S. 99.

44 Vgl. Byrne (1971). 
wodurch die Kooperation und letztlich die Performanz von Führungsteams beeinträchtigt werden können.

Erste empirische Hinweise für die moderierende Rolle der Teamgröße im Zusammenhang zwischen Gender Diversity und Performanz ergeben sich aus Untersuchungen in Nicht-Führungsteams. Wegge et al. (2008) konnten beispielsweise nachweisen, dass der Zusammenhang zwischen Gender Diversity und Performanz in großen Teams signifikant negativer ausfällt als in kleinen Teams ${ }^{45}$.

H1: Die Teamgröße moderiert den Zusammenhang zwischen Gender Diversity in Führungsteams und Unternehmensperformanz. So ist für kleinere Teams der Zusammenhang positiver als für größere Teams.

\subsubsection{Branche: Industrie vs. Dienstleistung}

Bei einer Branchendifferenzierung kann grundsätzlich zwischen Unternehmen im Dienstleistungs- und Produktionsbereich unterschieden werden ${ }^{46}$. Unternehmen aus diesen beiden Branchen können sich zum einen hinsichtlich ihrer Kundennähe und zum anderen in Bezug auf ihre geschlechterbezogene Beschäftigtenstruktur unterscheiden. Diese branchenspezifischen Eigenschaften können die Auswirkungen von Gender Diversity in Führungsteams beeinflussen.

Für die mit Gender Diversity verbundenen positiven Effekte im Sinne der Informationsund Entscheidungsperspektive kann sich insbesondere das Ausmaß der Kundennähe als relevant erweisen. So interagieren vor allem Unternehmen der Dienstleistungsbranche bei der Leistungserstellung intensiv mit den Konsumenten. Teilweise erfolgt der Konsum der erbrachten Leistungen sogar simultan mit deren Erstellung ${ }^{47}$. Dieser enge Kontakt erfordert ein hohes Maß an Sensibilität für die Bedürfnisse potenzieller Kunden ${ }^{48}$. Entsprechend kann im Sinne des Marktzugangsparadigmas ${ }^{49}$ und der Informations- und Entscheidungsperspektive Gender Diversity in Führungsteams von Dienstleistungsunternehmen als vorteilhafte Ressource verstanden werden. So spiegelt ein geschlechterdiverses Führungsteam tendenziell die Geschlechterheterogenität der potenziellen Kundschaft wider ${ }^{50}$. Entscheidungen innerhalb gemischt-geschlechtlicher Führungsteams können folglich besser auf die Bedürfnisse der Kundschaft abgestimmt werden ${ }^{51}$. Richard/Murthi/Ismail (2007) sprechen in einem ähnlichen Zusammenhang auch von einer erhöhten Marketingkompetenz, die es Unternehmen ermöglicht, Kunden anzu-

45 Vgl. Wegge et al. (2008).

46 Vgl. Jackson/Schuler/Rivero (1989), S. 730.

47 Vgl. Bowen/Schneider (1988); Grönroos (2006), S. 319.

48 Vgl. Jackson/Schuler/Rivero (1989), S. 730.

49 Vgl. Elmerich/Hornberger/Watrinet (2007), S. 22.

50 Vgl. Ali/Kulik/Metz (2011), S. 1470.

51 Vgl. Brammer/Millington/Pavelin (2009), S. 21. 
ziehen, zu binden und Veränderungen in den Kundenbedürfnissen zu erkennen ${ }^{52}$. Auch Unternehmen des Produktionsbereichs können prinzipiell von einem geschlechterdiversen Führungsteam profitieren, da auch hier dieses Potential besteht. Jedoch sind diese Unternehmungen in der Regel deutlich kapitalintensiver und in geringerem Maße auf eine direkte Kundenbeziehung angewiesen. Der Erfolg von Produktionsunternehmen ist daher grundsätzlich stärker von Ausstattung, Rohstoffen und Technologie abhängig ${ }^{53}$.

Für mögliche negative Effekte von Gender Diversity in Form sozialer Kategorisierungsprozesse könnte sich vor allem das branchenspezifische Geschlechterverhältnis als kritische Randbedingung erweisen ${ }^{54}$. Bestehende Untersuchungen zeigen, dass in Arbeitskontexten mit unausgeglichenem Geschlechterverhältnis oftmals Vorbehalte gegenüber des unterrepräsentierten Geschlechts vorherrschen ${ }^{55}$. Solche Stereotype können die Salienz des Diversity-Merkmals Geschlecht und damit verbundene soziale Kategorisierungsprozesse in Führungsteams beeinflussen. So wird die Salienz einer bestimmten sozialen Kategorie durch verschiedene Faktoren beeinflusst, wobei im Zusammenhang mit Gender Diversity insbesondere der so genannte „normative Fit" von Bedeutung ist ${ }^{56}$. Dieser beschreibt das Ausmaß, in dem das Geschlecht als soziale Kategorie für ein Individuum subjektiv Sinn ergibt und zu dessen Einstellungen, Erwartungen und Stereotypen passt. Branchenspezifische Geschlechterstereotypen erhöhen den normativen Fit und damit die Salienz des Merkmals Geschlecht, indem sie systematische arbeitsbezogene Unterschiede zwischen den Geschlechtern postulieren. Untersuchungen deuten darauf hin, dass in zahlreichen Ländern das Geschlechterverhältnis im Dienstleistungsbereich deutlich ausgeglichener ist als im produzierenden Gewerbe ${ }^{57}$. Entsprechend kann erwartet werden, dass im Dienstleistungsbereich Geschlechterstereotypen weniger verbreitet sind und das Merkmal Geschlecht folglich eine geringere Salienz besitzt; Gender Diversity in Führungsgremien von Dienstleistungsunternehmen sollte daher seltener zu sozialen Kategorisierungsprozessen führen als in Produktionsunternehmen.

Zusammenfassend lässt sich festhalten, dass für Dienstleistungsunternehmen ein positiverer Zusammenhang zwischen Gender Diversity in Führungsgremien und Unternehmensperformanz zu erwarten ist als für Unternehmen der Produktionsbranche. Hinweise darauf liefert auch die empirische Forschung: Brammer/Millington/Pavelin (2009) konnten beispielsweise nachweisen, dass sich die Präsenz von Frauen in Führungsgremien vor allem in Unternehmen mit engem Kontakt zum Endverbraucher positiv auf die Unternehmensreputation auswirkt ${ }^{58}$. Weiterhin konnten Ali/Kulik/Metz (2011) zeigen, dass der

52 Vgl. Richard/Murthi/Ismail (2007), S. 1219.

53 Vgl. Joshi/Roh (2009), S. 608f.; Richard/Murthi/Ismail (2007), S. 1220.

54 Vgl. Joshi/Roh (2009), S. 606f.

55 Vgl. Cabrera/Sauer/Thomas-Hunt (2009), S. 420; Fiske (1993), S. 622; Reskin/McBrier/Kmec (1999), S. 348.

56 Vgl. Turner et al. (1987), S. 117; Turner et al. (1994), S. 455.

57 Vgl. z.B. Brammer/Millington/Pavelin (2009), S. 21; Bundesagentur für Arbeit (2012), S. 13ff.; United States Census (2010).

58 Vgl. Brammer/Millington/Pavelin (2009). 
Dienstleistungsbereich stärker von Geschlechterheterogenität (bezogen auf die gesamtorganisationale Belegschaft) profitiert als der Produktionsbereich ${ }^{59}$.

H2: Die Branchenzugehörigkeit moderiert den Zusammenhang zwischen Gender Diversity in Führungsteams und Unternehmensperformanz. So ist für Unternehmen des Dienstleistungsbereichs der Zusammenhang positiver als für Unternehmen des Produktionsbereichs.

\subsubsection{Kultureller Kontext: Geschlechter-Egalitarismus}

Der kulturelle Kontext, in dem ein Unternehmen agiert, wurde in der Literatur wiederholt als kritische Randbedingung für Diversity-Effekte diskutiert ${ }^{60}$. In Bezug auf Gender Diversity dürfte dabei insbesondere das Ausmaß an Geschlechter-Egalitarismus im jeweiligen kulturellen Kontext eines Unternehmens von Bedeutung sein.

Geschlechter-Egalitarismus beschreibt das Ausmaß, in dem eine Gesellschaft Rollenunterschiede zwischen den Geschlechtern minimiert und gleichzeitig die Gleichberechtigung der Geschlechter betont ${ }^{61}$. In Gesellschaften mit hohem Geschlechter-Egalitarismus konvergieren die Geschlechterstereotypen und es wird eine Interaktion auf Augenhöhe zwischen den Geschlechtern angestrebt ${ }^{62}$. Das Ausmaß an Geschlechter-Egalitarismus kann sowohl die positiven Effekte im Sinne der Informations- und Entscheidungsperspektive als auch die negativen Effekte gemäß der sozialen Kategorisierungsperspektive in gemischtgeschlechtlichen Führungsteams beeinflussen.

Bezugnehmend auf die Informations- und Entscheidungsperspektive steht in geschlechterheterogenen Führungsteams durch die unterschiedlichen Fähigkeiten und Sichtweisen der männlichen und weiblichen Teammitglieder ein umfangreicher kognitiver Ressourcenpool zur Verfügung. Allerdings muss davon ausgegangen werden, dass dieser kognitive Ressourcenpool nicht automatisch genutzt wird, wie die heterogene Befundlage bestehender Studien indiziert ${ }^{63}$. In diesem Zusammenhang kann erwartet werden, dass die Informationsverarbeitung in geschlechterdiversen Führungsteams auch von den kulturell determinierten Werten und Einstellungen der Teammitglieder gegenüber den Geschlechtern abhängig ist. So dürften in kulturellen Kontexten mit stark ausgeprägtem Geschlechter-Egalitarismus die informationellen Ressourcen von Frauen als wertvoller und gewinnbringender erachtet werden als in Kulturen mit eher geringem GeschlechterEgalitarismus. Unter der Bedingung eines hohen Geschlechter-Egalitarismus sollten folglich Frauen in Führungsteams zum einen eher bereit sein, ihr Wissen und ihre Ansichten offen zu äußern; zum anderen sollten die informationellen Ressourcen von Frauen bei der

59 Vgl. Ali/Kulik/Metz (2011).

60 Vgl. z.B. Van der Vegt/Van de Vliert/Huang (2005).

61 Vgl. HouselJavidan (2004), S. 12.

62 Vgl. Emrich/Denmark/den Hartog (2004), S. $349 \mathrm{ff}$.

63 Vgl. z.B. Boerner/Keding/Hüttermann (2012). 
Entscheidungsfindung in Führungsteams stärker berücksichtigt werden ${ }^{64}$. Bei geringem Geschlechter-Egalitarismus hingegen wird das geschlechterspezifische Wissen von Frauen als weniger gewinnbringend erachtet und dürfte daher in geringerem Umfang in die Informationsverarbeitung eingehen.

Darüber hinaus kann das Ausmaß an Geschlechter-Egalitarismus in einer Kultur die Salienz des Diversity-Merkmals Geschlecht und damit verbundene soziale Kategorisierungsprozesse in Führungsteams beeinflussen. Wie in Kapitel 2.2.2 erörtert, kann das Ausmaß an Geschlechter-Egalitarismus den normativen Fit des Diversity-Merkmals Geschlecht erhöhen und dadurch Prozesse der sozialen Kategorisierung fördern. So kann der normative Fit einer sozialen Kategorie auch maßgeblich durch gesellschaftliche Normen und Werte determiniert werden ${ }^{65}$. Entsprechend weist das Diversity-Merkmal Geschlecht in Gesellschaften mit geringem Geschlechter-Egalitarismus, in denen Geschlechterstereotypen vorherrschen und Geschlechterunterschiede betont werden, einen hohen normativen Fit und somit eine hohe Salienz auf. Folglich sollte es in gemischt-geschlechtlichen Führungsteams eher zu sozialen Kategorisierungsprozessen und Subgruppenbildung basierend auf dem Geschlecht der Teammitglieder kommen. Hingegen ist in Gesellschaften, in denen ein hoher Geschlechter-Egalitarismus vorherrscht und dadurch die Geschlechterbilder stark überlappen, mit einem geringeren normativen Fit und einer entsprechend geringeren Salienz des Geschlechts zu rechnen. Soziale Kategorisierungsprozesse und Subgruppenbildung in gemischt-geschlechtlichen Führungsteams sollten dadurch weniger wahrscheinlich werden.

Zusammenfassend kann davon ausgegangen werden, dass in Kulturen mit hoch ausgeprägtem Geschlechter-Egalitarismus die möglichen positiven Effekte von Gender Diversity in Führungsteams gestärkt und gleichzeitig potenziell negative Effekte abgeschwächt werden. Empirisch wurde der kulturelle Kontext bislang noch nicht als Moderator des Zusammenhangs zwischen Gender Diversity in Führungsteams und Unternehmensperformanz untersucht. Zugleich liefert jedoch die Forschung zu Diversity-Klima ${ }^{66}$ und so genannten „Diversity Beliefs “67 einen empirischen Nachweis für die Annahme, dass Einstellungen gegenüber Diversity einen signifikanten Einfluss auf den Zusammenhang zwischen Diversity und organisationaler Effektivität besitzen ${ }^{68}$.

H3: Geschlechter-Egalitarismus moderiert den Zusammenhang zwischen Gender Diversity in Führungsteams und Unternehmensperformanz. So ist der Zusammenhang in Ländern mit hohem Geschlechter-Egalitarismus positiver als in Ländern mit geringerem Geschlechter-Egalitarismus.

64 Vgl. Homan et al. (2007), S. 1190.

65 Vgl. Pearsall/Ellis/EVans (2008), S. 227; Van Knippenberg/De Dreu/Homan (2004), S. 1014. Komplementär zur Argumentation in Kapitel 2.2.2 kann davon ausgegangen werden, dass Geschlechterstereotypen sowohl von branchen- als auch von kulturspezifischen Rahmenbedingungen abhängig sind (vgl. Stelter (2002), S. 93ff.).

66 Vgl. z.B. Kunze/Boehm (2013), S. $51 \mathrm{ff}$.

67 Vgl. z.B. Homan et al. (2010).

68 Vgl. Van Knippenberg/Haslam (2003). 


\subsubsection{Performanzkriterium: Finanzielle Erfolgskennzahlen vs. Innovativität}

Die Verwendung unterschiedlicher Performanzkriterien könnte ebenfalls für die Heterogenität der Befunde zum Zusammenhang zwischen Gender Diversity in Führungsteams und Unternehmensperformanz von Bedeutung sein. So kann die Performanz eines Unternehmens zum einen im Sinne finanzieller Erfolgskennzahlen als Rentabilität und Ertrag eines Unternehmens verstanden werden ${ }^{69}$. Zum anderen kann aber auch die Innovativität eines Unternehmens als Performanzmaß herangezogen werden ${ }^{70}$.

In Anlehnung an die Informations- und Entscheidungsperspektive wird erwartet, dass Gender Diversity in Führungsteams insbesondere im Hinblick auf die Innovativität von Relevanz ist. Denn der mit Gender Diversity einhergehende vergrößerte Wissenspool und die Konfrontation mit bislang unbeachteten Sachargumenten und Perspektiven kann die Entwicklung neuer und innovativer Ideen in Führungsteams fördern ${ }^{71}$. Zugleich kann die kritische Auseinandersetzung mit verschiedenen Sichtweisen und die Vielfalt an Erfahrungshintergründen dazu führen, dass die praktische Realisierbarkeit neuer Ideen umfassender beurteilt wird ${ }^{72}$. Dies kann beispielweise bedeuten, dass gemischtgeschlechtliche Führungsteams eher die Potentiale neuer Märkte, Prozesse und Produkte erkennen und als sogenannte „Innovation Champions“ die Umsetzung von besonders vielversprechenden Ideen sicherstellen ${ }^{73}$.

Im Gegensatz dazu ist für den Zusammenhang zwischen Gender Diversity in Führungsteams und finanziellen Erfolgskennzahlen eine weniger enge theoretische Verbindung zu erwarten. So wird der Effekt von Diversity auf die finanzielle Unternehmensperformanz oftmals über eine gesteigerte Innovationsleistung begründet ${ }^{74}$. Frühere Forschung hat allerdings gezeigt, dass die Innovationsleistung und die finanzielle Performanz eines Unternehmens nicht unmittelbar zusammenhängen, sondern dieser Zusammenhang von weiteren Randbedingungen abhängig ist ${ }^{75}$.

Dementsprechend ist zu vermuten, dass positive Effekte von Gender Diversity in Führungsteams insbesondere dann zu erwarten sind, wenn Innovativität und nicht finanzielle Performanz das Performanzkriterium bildet. Im Einklang mit dieser Erwartung konnten Miller/Triana (2009) einen signifikant positiven Effekt von Gender Diversity in Führungsgremien auf Innovationsleistung nachweisen, nicht jedoch auf die finanzielle Performanz eines Unternehmens ${ }^{76}$.

69 Vgl. z.B. Simons/Pelled/Smith (1999), S. 664.

70 Vgl. z.B. West/Anderson (1996); S. 680ff.

71 Vgl. Van Dijk/Van Engen/Van Knippenberg (2012), S. 41; Van Knippenberg/Schippers (2007), S. 518.

72 Vgl. Gibson (2001), S. 126.

73 Vgl. Chakrabarti (1974), S. 59; Elenkov/Manev (2005), S. 382; Miller/Triana (2009), S. $759 f f .$.

74 Vgl. z.B. Dezsö/Ross (2012), S. 1077; Miller/Triana (2009), S. 764.

75 Vgl. Baer/Frese (2003).

76 Vgl. Miller/Triana (2009). 
H4: Das Performanzkriterium moderiert den Zusammenhang zwischen Gender Diversity in Führungsteams und Unternehmensperformanz. So ist der Zusammenhang mit innovationsbezogenen Kriterien positiver als mit finanziellen Erfolgskennzahlen.

\section{Methode}

\subsection{Literaturrecherche und Einschlusskriterien}

Um eine möglichst vollständige Erfassung der bestehenden empirischen Literatur zum Zusammenhang zwischen Gender Diversity in Führungsteams und Unternehmensperformanz sicherzustellen, wurden vier Suchstrategien kombiniert. Erstens wurden die einschlägigen Fachdatenbanken (Business Source Premier, PsycArticles, PsycINFO, Social Science Citation Index und WisoNet) nach themenbezogener Literatur für den Zeitraum von 1980 bis einschließlich Juli 2013 durchsucht $^{77}$. Für die Suche wurden die Begriffe Gender Diversity, Gender Heterogeneity, Gender Composition, Performance, Innovation, Creativity und Effectiveness in unterschiedlicher Weise verknüpft. Zusätzlich wurde in diesem Schritt mittels der Suchmaschinen „Google“ und "Google Scholar" nach unveröffentlichter Literatur gesucht. Zweitens wurden die Literaturverzeichnisse bestehender Metaanalysen ${ }^{78}$ und Review-Artikel ${ }^{79}$ im Bereich Diversity auf weitere relevante Artikel durchsucht. Drittens wurden Aufrufe über Mailing-List-Server der Academy of Management versendet mit der Bitte um Zusendung unveröffentlichter Studien. Viertens wurden die durch die ersten drei Suchstrategien identifizierten Studien systematisch nach Referenzen zu weiteren relevanten Artikeln durchsucht (so genanntes „Schneeballverfahren“).

Die so identifizierten Einzelstudien wurden in der Metaanalyse berücksichtigt, wenn sie die nachfolgenden fünf Einschlusskriterien erfüllten: Erstens musste Gender Diversity für Führungsgremien berichtet werden. Dies bedeutet, dass in den Studien explizit die Geschlechterheterogenität des Aufsichtsrats, des Vorstandes oder beider Gremien gemessen werden musste ${ }^{80}$. Zweitens musste finanzielle Performanz oder Innovativität auf Organisationsebene berichtet werden. Drittens waren ausreichende statistische Informationen (d.h. Stichprobengröße und Korrelationskoeffizienten) in den Studien erforderlich, um

77 Dem umfassenden Literaturüberblick der Diversity-Forschung von Williams/O'Reilly (1998) zufolge wurden die ersten empirischen Analysen zu den Auswirkungen von Diversity in Führungsgremien (Top Management Teams) Ende der 1980er Jahre veröffentlicht (vgl. z.B. Bantel/Jackson (1989); Murray (1989)). Entsprechend wurde als Beginn des Suchzeitraums das Jahr 1980 gewählt.

78 Vgl. Bell et al. (2011); Bowers/Pharmer/Salas (2000); Horwitz/Horwitz (2007); Joshi/Roh (2009); Stewart (2006); Van Dijk/Van Engen/Van Knippenberg (2012); Webber/Donahue (2001).

79 Vgl. Boerner/Keding/Hüttermann (2012); Jackson/Joshi (2011); Van Knippenberg/Schippers (2007).

80 Ursprünglich war intendiert, die Art des untersuchten Führungsgremiums (d.h. Leitungs- oder Kontrollgremium) ebenfalls als Moderator in der Analyse zu berücksichtigen; allerdings ging die Art des untersuchten Gremiums aus den Beschreibungen der meisten Einzelstudien nicht klar hervor. Verantwortlich hierfür dürfte u.a. sein, dass in vielen Ländern (z.B. im US-amerikanischen Kontext) oftmals keine explizite Trennung zwischen dem Leitungs- und Kontrollgremium eines Unternehmens existiert (vgl. Holst/Kirsch (2014), S.27). Daher musste auf die Untersuchung dieses Moderators verzichtet werden. 
die entsprechenden Effektgrößen für die Metaanalyse berechnen zu können. Viertens wurde davon Abstand genommen, mehrere Studien derselben Autoren aufzunehmen, wenn diese auf einem identischen Datensatz basierten. In diesem Fall wurde nur die Studie berücksichtigt, welche die größere Zahl an interessierenden Variablen berichtete. Fünftens konnten nur solche Studien in Betracht gezogen werden, die entweder in englischer oder in deutscher Sprache verfasst sind ${ }^{81}$.

Nach Anwendung dieser Kriterien besteht der finale Datensatz aus 38 Einzelstudien, die im Literaturverzeichnis mit einem Asterisk gekennzeichnet sind. Alle berücksichtigten Studien wurden im Zeitraum zwischen 1997 und 2013 durchgeführt und untersuchten insgesamt 10.664 Führungsteams.

\subsection{Kodierung der Studien}

Alle berücksichtigten Einzelstudien wurden jeweils unabhängig von mindestens zwei Autoren dieses Beitrags kodiert. Die Interrater-Übereinstimmung war hoch, wobei die kodierten Informationen zu Stichprobengrößen, Effektstärken und Moderatoren in 94,1\% der Fälle übereinstimmten ${ }^{82}$. Die bestehenden Diskrepanzen in der Kodierung wurden mittels Diskussion behoben.

Die Informationen zu den in Kapitel 2.2 erörterten theoretischen Moderatoren wurden wie folgt kodiert: (a) Zur Erfassung der Teamgröße wurde ein Mediansplit der z-transformierten Teamgrößenvariable durchgeführt, wodurch sich eine Subgruppe mit kleinen und eine mit großen Führungsteams ergab ${ }^{83}$. (b) Informationen zur Branche wurden kodiert, sofern die Einzelstudien diese berichteten. Studien wurden entweder dem Dienstleistungsbereich,

81 Basierend auf diesen Kriterien wurden Studien ausgeschlossen, die Gender Diversity in Teams auf niedrigeren Hierarchieebenen oder für gesamte Organisationen ausweisen. Des Weiteren wurden Studien nicht berücksichtigt, die Faultlines (vgl. Kapitel 5.2) erfassen oder mehrere Diversity-Attribute auf andere Art zu einer gemeinsamen Maßzahl zusammenfassen, sodass kein Rückschluss auf die spezifische Wirkung von Gender Diversity möglich ist. Ferner wurden Studien ausgeschlossen, die Performanz auf der Individual- und Teamebene messen oder lediglich nicht-finanzielle Kennzahlen (z.B. soziale Performanz) untersuchen. Den Ausführungen von Hunter/Schmidt (2007, S. 192ff., vgl. auch Lipsey/Wilson, (2007), S. 15f.) folgend wurden überdies Studien nicht berücksichtigt, die als einziges Zusammenhangsmaß Regressionskoeffizienten berichten, da diese eine mangelnde Vergleichbarkeit über die einzelnen Studien hinweg aufweisen. Außerdem wurden Studien mit fehlenden Angaben zu ihrer Stichprobengröße ausgeschlossen, da dadurch keine Korrektur von Stichprobenfehlern möglich ist. Um den Ausschluss von Studien aufgrund dieser fehlenden Angaben zu reduzieren, wurden die Autoren der entsprechenden Studien mit der Bitte um Bereitstellung der notwendigen Informationen kontaktiert.

82 Vgl. Allen et al. (2004), S. 129; de Wit/Greer/Jehn (2012), S. 365.

83 Für eine vergleichbare Vorgehensweise siehe z.B. die Metaanalyse von Stahl et al. (2010). 
dem Produktionsbereich oder einer Querschnittskategorie aus beidem zugeordnet ${ }^{84}$. Die Kodierung ergab, dass lediglich zwei Einzelstudien ausschließlich Produktionsunternehmen untersuchen. Da auf Basis einer so geringen Zahl von Studien keine validen Ergebnisse bei einer Moderationsanalyse zu erwarten sind, kann im Folgenden lediglich zwischen Dienstleistungsunternehmen und einer Querschnittskategorie aus Dienstleistungs- und Produktionsunternehmen differenziert werden; entsprechend ist eine exakte Überprüfung von Hypothese 2 nicht möglich (vgl. Kapitel 4). (c) Zur Erfassung des kulturellen Kontexts wurde das Land, in dem eine Studie durchgeführt wurde, auf der Geschlechter-Egalitarismus-Dimension der GLOBE-Studie (Global Leadership and Organizational Behavior Effectiveness ${ }^{85}$ ) verortet; die GLOBE-Studie stellt den derzeit aktuellsten und umfassendsten Ansatz eines dimensionsbasierten Kulturvergleichs dar ${ }^{86}$. Die Verortung erfolgte durch die Zuordnung zu einer von drei Klassen von Geschlechter-Egalitarismus-Werten, die in der GLOBE-Studie unterschieden werden ${ }^{87}$. Dabei bestehen signifikante Unterschiede zwischen, jedoch nicht innerhalb der drei Klassen. Entsprechend wurden die Einzelstudien gemäß dem Land, in dem sie durchgeführt wurden, der Klasse geringer, mittlerer oder hoher Geschlechter-Egalitarismus-Werte zugeordnet. (d) Ferner wurden Informationen zum verwendeten Performanzkriterium erfasst. Dabei wurden die Effektgrößen zwischen Gender Diversity und Gesamtkapitalrentabilität (Return on Assets bzw. ROA), Eigenkapitalrentabilität (Return on Equity bzw. ROE), Umsatzrentabilität (Return on Sales bzw. ROS), Buch-zu-Markt-Wert (Tobin's Q), Nettobetriebseinkommen, Gewinn pro Angestelltem, Anstieg des Aktienkurses, Gewinn pro Aktie und et cetera in der Subgruppe finanzieller Erfolgskennzahlen zusammengefasst. Für das Performanzkriterium Innovation wurden $\mathrm{Zu}$ sammenhänge von Gender Diversity mit der Anzahl der Patente sowie mit den Investitionen in Forschung und Entwicklung klassifiziert ${ }^{88}$.

Zusätzlich wurden folgende methodische Merkmale der Einzelstudien erfasst, die ebenfalls für die heterogene Befundlage der bestehenden Forschung verantwortlich sein könnten

84 In Übereinstimmung mit bisheriger Forschung (vgl. Ali/Kulik/Metz (2011); Richard/Murthi/Ismail (2007)) wurden die nach der Standard Industrial Classification (vgl. United States Department of Labor (1987)) unterschiedenen Industriegruppen Transport, Kommunikation, Energieversorgung, Wasser- und Abwasserversorgung, Großhandel, Einzelhandel, Finanzen, Versicherungen, Immobilien und eine Sammelkategorie an weiteren Serviceunternehmen zum Dienstleistungsbereich zusammengefasst. Unter dem Produktionsbereich wurden hingegen die Industriegruppen Agrarwirtschaft, Forstwirtschaft, Fischerei, Bergbau, Baugewerbe und weitere Produktionsunternehmen subsumiert (für detailliertere Definitionen der einzelnen Industriegruppen siehe United States Department of Labor (1987)).

85 Vgl. House et al. (2004). In der GLOBE-Studie wird Geschlechter-Egalitarismus nochmals in eine Einstellungskomponente und eine Verhaltenskomponente untergliedert. In Anbetracht der diesem Beitrag zugrundeliegenden sozialpsychologischen Theorien und den bisherigen Befunden der Diversity-Forschung zur Zentralität von Einstellungen (vgl. Homan et al. (2007); Van Dick et al. (2008); Van Knippenberg/Haslam/Platow (2007)) erfolgt eine ausschließliche Fokussierung auf die Einstellungskomponente.

86 Vgl. Engelen/Brettel (2011), S. 519.

87 Vgl. Emrich/Denmark/den Hartog (2004), S. 366.

88 Die Forschungs- und Entwicklungsausgaben stellen dabei zwar lediglich einen Proxy für die tatsächliche Innovationsleistung eines Unternehmens dar; sie kommen jedoch aufgrund der hohen Korrelation mit anderen Innovationsindikatoren in der strategischen Managementforschung häufig zur Anwendung (vgl. Balkin/Markman/ Gomez-Mejia (2000); Hagedoorn/Cloodt (2003)). 
(vgl. Abbildung 1): (a) In Bezug auf das Studiendesign wurde zwischen Quer- und Längsschnittuntersuchungen unterschieden. Studien, welche die unabhängige und abhängige Variable zum gleichen Zeitpunkt $t$ erhoben, wurden als Querschnittanalysen eingestuft. Wurde die unabhängige Variable zum Zeitpunkt $t$ und die abhängige zum Zeitpunkt $t+1$ erhoben oder ein Paneldesign verwendet, wurde die Untersuchung als Längsschnittstudie kodiert. (b) Bei der Operationalisierung von Gender Diversity wurde zwischen dem Index nach Blau (1977), dem prozentualen Frauenanteil, einer dichotomen Messung ( 0 = keine Frau im Führungsteam; 1 = mindestens eine Frau) und der absoluten Anzahl weiblicher Teammitglieder unterschieden ${ }^{89}$. (c) Hinsichtlich der Operationalisierung der finanziellen Unternehmensperformanz (vgl. Kapitel 2.2.4) wurde bei der Kodierung zusätzlich nach bilanzbasierten und aktienbasierten Kennzahlen unterschieden. In Anlehnung an bestehende Arbeiten wurden die Maße ROA, ROE, ROS und Gewinn pro Angestellter als bilanzbasierte Kennzahlen klassifiziert ${ }^{90}$. Maße wie Tobin's Q, Anstieg des Aktienkurses und Gewinn pro Aktie wurden hingegen als aktienbasierte Kennzahlen eingestuft. (d) Abschließend wurde noch der Publikationsstatus erhoben, das heißt die Unterscheidung zwischen veröffentlichten und unveröffentlichten Studien.

Keine dieser zusätzlich erhobenen Studieninformationen stellte einen signifikanten Moderator des Zusammenhangs zwischen Gender Diversity in Führungsteams und Unternehmensperformanz dar. Aus diesem Grund wird auf die Analyseergebnisse zu methodischen Moderatoren im Folgenden nicht weiter eingegangen ${ }^{91}$.

\subsection{Metaanalytisches Vorgehen zur Hypothesenprüfung}

In dieser Studie wurde das metaanalytische Verfahren nach Hunter/Schmidt (2007) verwendet, das insgesamt vier Schritte umfasst ${ }^{92}$. Entsprechend wurden im ersten Schritt die Effekte in den Einzelstudien für mögliche Artefakte korrigiert ${ }^{93}$. Zu den am häufigsten durchgeführten Artefaktkorrekturen gehören die Bereinigung der Stichprobenfehler und die Behebung von Messfehlern der abhängigen und unabhängigen Variablen. In der vorliegenden Analyse wurde lediglich für Stichprobenfehler korrigiert, indem jede Korrelation mit der Stichprobengröße der jeweiligen Einzelstudie gewichtet wurde. Die Korrektur von Messfehlern war hingegen nicht möglich, weil hierfür Reliabilitätsmaße der abhängigen und unabhängigen Variablen erforderlich sind. Da jedoch weder für Gender Diversity noch für Indikatoren der Unternehmensperformanz Reliabilitätsmaße berechnet werden können, waren in den Einzelstudien keine entsprechenden Informationen enthalten.

89 Vgl. Blau (1977).

90 Vgl. Abdullah/Ismail/Nachum (2012); Haslam et al. (2010).

91 Die entsprechenden Analyseergebnisse können jedoch auf Anfrage durch die Autoren zur Verfügung gestellt werden.

92 Vgl. Hunter/Schmidt (2007).

93 Vgl. Hunter/Schmidt (2007), S. $33 \mathrm{ff}$. 
Im zweiten Schritt wurde der direkte Effekt geschätzt. Dazu wurde aus den für die Stichprobenfehler korrigierten Korrelationen die mittlere gewichtete Korrelation $(\rho)$ berechnet. Um die statistische Unabhängigkeit der einzelnen Effektgrößen zu gewährleisten, wurde bei der Bildung des Mittelwertes aus jeder Einzelstudie nur eine Effektgröße berücksichtigt ${ }^{94}$. Einige Einzelstudien berichten konzeptuelle Replikationen (z.B. verschiedene Performanzkriterien oder Operationalisierungen von Gender Diversity). In diesem Fall wurde aus den einzelnen Korrelationen ein Mittelwert gebildet, bevor diese in der Analyse berücksichtigt wurden.

Im dritten Schritt erfolgte die Berechnung des Standardfehlers der mittleren gewichteten Korrelation $\left(\mathrm{SF}_{\rho}\right)$. Außerdem wurden das $95 \%$ Konfidenzintervall (KI) und das $90 \%$ Kredibilitätsintervall (KV) um die mittlere gewichtete Korrelation berechnet. Schließt das 95\% KI den Wert Null nicht mit ein, ist dies ein Hinweis auf einen auf dem 5\%-Niveau signifikanten Gesamteffekt ${ }^{95}$. Das 90\% KV liefert Hinweise auf die Heterogenität des Gesamteffekts. Ist das $90 \% \mathrm{KV}$ relativ groß oder schließt den Wert Null mit ein, wird dies als Hinweis auf mögliche Moderatoren gesehen ${ }^{96}$. Gleichzeitig spricht ein KV, das den Wert Null umfasst, gegen die Generalisierbarkeit der mittleren gewichteten Korrelation $(\rho)$. Um sicherzustellen, dass die in der Metaanalyse errechnete Effektstärke und deren Heterogenität nicht maßgeblich durch wenige Ausreißer beeinflusst werden, wurde zusätzlich die Sample-Adjusted Meta-Analytic Deviance (SAMD) Statistik nach Huffcut/Arthur (1995) berechnet ${ }^{97}$.

Im vierten Analyseschritt wurde die Wirkung der Moderatoren überprüft. Dabei wurden die Studien nach dem entsprechenden kategorialen Moderator in Subgruppen zusammengefasst. Waren die in Schritt zwei angesprochenen konzeptionellen Replikationen für die Moderationsanalyse relevant (d.h. es wurden beispielsweise in einer Studie sowohl Korrelationen mit finanzieller Performanz als auch mit Innovation berichtet), wurden diese getrennt voneinander den jeweiligen Subgruppen zugewiesen. Anschließend wurden die Analyseschritte zwei und drei erneut für die einzelnen Subgruppen durchgeführt. Überlappen die 95\% KI für die Effekte in den Subgruppen nicht, wird dies als Indiz für die Existenz eines Moderationseffekt gewertet ${ }^{98}$. Zusätzlich dazu wurde die Wirkung der kontinuierlichen Moderatorvariablen Teamgröße und Geschlechter-Egalitarismus mittels einer Weighted Least Squares (WLS) Regression überprüft"9 . Wie von Lipsey/Wilson (2011) vorgeschlagen, wurden dabei die Effektgrößen der Einzelstudien als Kriteriumsvariable und die kontinuierlichen Moderatorvariablen als Prädiktor in das Regressionsmodell eingeführt, wobei jede Effektgröße durch die Inverse ihrer Varianz gewichtet wur-

94 Vgl. Hunter/Schmidt (2007), S. 430ff.

95 Vgl. Hunter/Schmidt (2007), S. 205ff.

96 Vgl. Hunter/Schmidt (2007), S. 205ff.; Whitener (1990).

97 Vgl. Huffcut/Arthur (1995); Drei Studien wurden als Ausreißer identifiziert (Krishnan/Park (2005); Rambo (2013); Roh (2010)); eine umfassende Inspektion dieser Einzelstudien lieferte allerdings keine Hinweise auf ungewöhnliche Studieneigenschaften, die extreme Effektgrößen hervorrufen könnten. Zudem hatte ein Ausschluss dieser Studien keinen signifikanten Einfluss auf die Ergebnisse der Analyse. Daher wurde bei der nachfolgenden Analyse auf deren Ausschluss verzichtet.

98 Vgl. Hunter/Schmidt (2007), S. 423f.

99 Vgl. Hedges/Olkin (1985), S. 167ff.; Viechtbauer (2007), S. $110 \mathrm{ff}$. 
$\mathrm{de}^{100}$. Es wurden dabei sowohl konservativere Mixed-Effects-Modelle als auch weniger konservative Fixed-Effects-Modelle berechnet ${ }^{101}$.

\section{Ergebnisse}

Wie aus Tabelle 1 ersichtlich, besteht ein äußerst schwacher positiver Zusammenhang zwischen Gender Diversity in Führungsteams und Unternehmensperformanz insgesamt ( $\rho=0,016)$, jedoch schließt das $95 \%$ KI den Wert Null mit ein. Folglich existiert im Mittel kein signifikanter Zusammenhang zwischen einer gemischtgeschlechtlichen Zusammensetzung von Führungsteams und Unternehmensperformanz insgesamt. Wie dem 90\% KV zu entnehmen ist, weisen die Einzelstudien zudem eine beträchtliche Heterogenität hinsichtlich der Effektstärken auf, was auf die Existenz moderierender Variablen hinweist. Die Ergebnisse der Moderationsanalysen werden im Folgenden berichtet.

Tabelle 1: Metaanalytische Ergebnisse des Zusammenhangs zwischen Gender Diversity und Unternehmensperformanz insgesamt sowie der Subgruppenanalysen

\begin{tabular}{lcccccccccc}
\hline & $k$ & $N$ & $r$ & $\rho$ & $S A$ & $S F \rho$ & $90 \% \mathrm{KV}$ & $95 \% \mathrm{KI}$ \\
\hline $\begin{array}{l}\text { Gender Diversity } \\
\text { Größe des Führungsteams }\end{array}$ & 46 & 10.664 & 0,035 & 0,016 & 0,054 & 0,013 & $-0,074$ & 0,105 & $-0,010$ & 0,042 \\
Klein & 14 & 4.202 & 0,040 & 0,018 & 0,030 & 0,017 & 0,032 & 0,068 & $-0,016$ & 0,052 \\
Groß & 14 & 3.347 & 0,061 & $-0,015$ & 0,032 & 0,019 & $-0,068$ & 0,037 & $-0,053$ & 0,023 \\
$\begin{array}{l}\text { Branche } \\
\text { Dienstleistung }\end{array}$ & 7 & 737 & 0,085 & $-0,060$ & 0,129 & 0,061 & $-0,272$ & 0,152 & $-0,180$ & 0,060 \\
Gemischt & 31 & 9.927 & 0,024 & 0,021 & 0,039 & 0,012 & $-0,042$ & 0,085 & $-0,002$ & 0,045 \\
$\begin{array}{l}\text { Geschlechter-Egalitarismus } \\
\text { Gering }\end{array}$ & & & & & & & & & & \\
Mittel & 4 & 1.590 & $-0,007$ & 0,007 & 0,024 & 0,028 & $-0,032$ & 0,047 & $-0,047$ & 0,062 \\
Hoch & 26 & 8.542 & 0,006 & 0,013 & 0,052 & 0,015 & $-0,071$ & 0,098 & $-0,016$ & 0,042 \\
$\begin{array}{l}\text { Performanzkriterium } \\
\text { Finanzielle }\end{array}$ & & & & & & & & & & \\
Erfolgskennzahlen & 38 & 10.664 & 0,038 & 0,027 & 0,058 & 0,014 & $-0,068$ & 0,123 & 0,001 & 0,054 \\
Innovation & 8 & 4.662 & $-0,008$ & $-0,042$ & 0,076 & 0,031 & $-0,167$ & 0,083 & $-0,102$ & 0,018 \\
\hline
\end{tabular}

Anmerkungen: $k=$ Anzahl der Effektstärken; $N=$ Gesamtstichprobengröße über alle Studien hinweg, basierend auf der Anzahl der untersuchten Führungsteams; $\rho=$ Mittlere ungewichtete Korrelation; $\rho=$ Mittlere gewichtete Korrelation; $S A_{\rho}=$ Standardabweichung von $\rho ; S F_{\rho}=$ Asymptotischer Standardfehler von $\rho$; $90 \% \mathrm{KV}=90 \%$ Kredibilitätsintervall; $95 \% \mathrm{KI}=95 \%$ Konfidenzintervall. 
Teamgröße. Laut Hypothese 1 ist für kleinere Führungsteams ein positiverer Zusammenhang zwischen Gender Diversity und Unternehmensperformanz zu erwarten als für größere Teams. Empirisch lässt sich in der Tat ein negativer Zusammenhang für große Führungsteams $(\rho=-0,015)$ und ein positiver Zusammenhang für kleine Teams $(\rho=0,018)$ zeigen (vgl. Tabelle 1). Allerdings überlappen die KI zwischen den beiden Subgruppen, weshalb Hypothese 1 als nicht bestätigt angesehen werden muss. Dieser Befund deckt sich auch mit den Ergebnissen der WLS-Regression ${ }^{102}$.

Branche. Hypothese 2 prognostiziert, dass Dienstleistungsunternehmen im Vergleich zu Produktionsunternehmen stärker von Gender Diversity in Führungsteams profitieren. Wie in Kapitel 3.2 dargelegt, können aufgrund der Datenlage lediglich Untersuchungen von Dienstleistungsunternehmen mit Studien verglichen werden, die einen Querschnitt aus Dienstleistungs- und Produktionsunternehmen analysieren. In Anlehnung an $\mathrm{Hy}-$ pothese 2 kann dabei vermutet werden, dass der Zusammenhang zwischen Gender Diversity und Unternehmensperformanz in der Dienstleistungskategorie positiver ausfällt als in der Querschnittskategorie. Diese Vermutung lässt sich allerdings nicht bestätigen (vgl. Tabelle 1): So ergibt die Analyse keinen signifikanten Unterschied zwischen dem leicht positiven Zusammenhang in der Querschnittskategorie $(\rho=0,021)$ und dem sogar schwach negativen Effekt in der Dienstleistungskategorie $(\rho=-0,060)$.

Kultureller Kontext. In Hypothese 3 wurde erwartet, dass Gender Diversity positiver mit Unternehmensperformanz verbunden ist, wenn das Ausmaß an Geschlechter-Egalitarismus in einem Land hoch ist. Wie die Ergebnisse in Tabelle 1 zeigen, sind die mittleren korrigierten Korrelationen zwischen Gender Diversity und Unternehmensperformanz sowohl bei geringem $(\rho=0,007)$ als auch bei mittlerem $(\rho=0,021)$ und hohem Gender Egalitarismus $(\rho=0,013)$ schwach positiv. Dabei ist entgegen der Annahme der $\mathrm{Zu}$ sammenhang für die Subgruppe mit mittlerem Geschlechter-Egalitarismus am höchsten, auch wenn der Effekt bei geringem Geschlechter-Egalitarismus wie erwartet am geringsten ausfällt. Diese Unterschiede in den Effektstärken zwischen den Subgruppen sind allerdings aufgrund der überlappenden 95\% KI nicht signifikant. Die zusätzlich durchgeführte WLS Regression mit der kontinuierlichen Geschlechter-Egalitarismus-Variable kommt zum gleichen Ergebnis ${ }^{103}$. Insgesamt ist Hypothese 3 demnach zurückzuweisen ${ }^{104}$.

102 Sowohl im Mixed-Effects-Modell $(\beta=-0,003, p=0,716)$ als auch im Fixed-Effects-Modell $(\beta=-0,004, p=$ $0,451)$ konnte die Teamgröße nicht als Moderator bestätigt werden.

103 Weder im Mixed-Effects-Modell $(\beta=-0,002, p=0,950)$ noch im Fixed-Effects $(\beta=-0,001, p=0,979)$ konnte Geschlechter-Egalitarismus als Moderator bestätigt werden.

104 Neben Geschlechter-Egalitarismus wurde auch die moderierende Rolle der im GLOBE-Projekt enthaltenen Kulturdimension „Gesellschaftlicher Kollektivismus" (vgl. House et al. (2004)) empirisch überprüft. Für heterogen zusammengesetzte Nicht-Führungsteams konnte Kollektivismus bereits als einflussreiche Randbedingung nachgewiesen werden (vgl. z.B. Chatman et al. (1998); Chatman/Spataro (2005)). Dieses Ergebnis konnte für die Führungsteams in der vorliegenden Analyse jedoch nicht repliziert werden. So bestanden keine signifikanten Unterschiede in den Zusammenhängen bei gering, mittel und hoch ausgeprägtem gesellschaftlichem Kollektivismus. 
Verwendetes Performanzkriterium. Im Rahmen von Hypothese 4 wurde vermutet, dass Gender Diversity in Führungsteams insbesondere dann von Vorteil ist, wenn Innovation und nicht finanzielle Erfolgskennzahlen das Performanzkriterium bildet. Empirisch lässt sich für diese Hypothese aber kein Beleg finden: Entgegen der Erwartung ist die Verbindung von Gender Diversity mit Innovation leicht negativ $(\rho=-0,042)$ und mit finanziellen Erfolgskennzahlen leicht positiv $(\rho=0,027)$; aufgrund der stark überlappenden Konfidenzintervalle kann dabei kein Beleg für signifikante Unterschiede zwischen den Effektstärken gefunden werden, was gegen die Existenz eines Moderationseffekts spricht. Jedoch tritt durch die Subgruppenanalyse ein anderer interessanter Befund zutage: So erweist sich der geringe positive Effekt von Gender Diversity auf finanzielle Erfolgskennzahlen als signifikant, auch wenn das relativ breite und die Null umfassende KV gegen die Generalisierbarkeit dieses Effekts spricht (vgl. Tabelle 1).

\section{Diskussion}

Sowohl die theoretischen Vorhersagen als auch die bestehenden empirischen Befunde zum Zusammenhang zwischen einer gemischtgeschlechtlichen Zusammensetzung von Führungsteams und Unternehmensperformanz sind widersprüchlich. Vor diesem Hintergrund verfolgte der Beitrag das Ziel einer umfassenden Metaanalyse zu der Frage, wie und in Abhängigkeit von welchen Randbedingungen Gender Diversity in Führungsteams mit Unternehmensperformanz verbunden ist. Auf Basis von 38 Einzelstudien, die insgesamt 10.664 Führungsteams untersuchen, kommt die vorliegende Analyse zu folgenden Ergebnissen: Erstens kann kein signifikanter Zusammenhang zwischen Gender Diversity in Führungsteams und Unternehmensperformanz insgesamt identifiziert werden. Zweitens können weder die Größe des Führungsteams noch die Branche, der kulturelle Kontext oder das verwendete Performanzkriterium als signifikante Moderatoren des Zusammenhangs bestätigt werden. Als zusätzlicher Befund aus der Moderationsanalyse ergab sich jedoch, dass Gender Diversity offenbar einen - wenn auch sehr kleinen - signifikant positiven Effekt auf finanzielle Erfolgskennzahlen besitzt; die Variabilität der entsprechenden Effektstärken in den Einzelstudien erlaubt aber keine Generalisierbarkeit dieses Ergebnisses über verschiedene Kontexte hinweg.

\subsection{Theoretische Integration der Ergebnisse}

Der Zusammenhang zwischen Gender Diversity in Führungsteams und Unternehmensperformanz insgesamt (bestehend aus finanziellen Erfolgskennzahlen und Innovativität) ist in der vorliegenden Analyse äußerst schwach $(\rho=0,016)$ und nicht signifikant. Dabei ist der Effekt in seiner absoluten Höhe vergleichbar mit den Ergebnissen anderer Metaanalysen, die entweder ausschließlich Nicht-Führungsteams ${ }^{105}$ oder eine Mischung 
aus Nicht-Führungsteams und Führungsteam $s^{106}$ untersuchen. Allerdings erwies sich der Zusammenhang in früheren Metaanalysen als schwach negativ sowie teilweise signifikant und änderte sich unter Einbezug bestimmter Moderatoren in Höhe und/oder Richtung. Das Ergebnis der vorliegenden Analyse spricht somit für die Vermutung, dass die Forschungsergebnisse zu Gender Diversity in Nicht-Führungsteams nur bedingt auf Führungsgremien übertragen werden können.

Zugleich ist der nicht signifikante Zusammenhang zwischen Gender Diversity und Unternehmensperformanz insgesamt auch aus der Perspektive der Diversity-Forschung allgemein interessant. So herrschte dort lange Zeit die Sichtweise vor, dass beziehungsorientierte Diversity-Merkmale wie Geschlecht ausschließlich negative Effekte in Form von sozialen Kategorisierungsprozessen hervorrufen; mit aufgabenorientierten DiversityMerkmalen wie dem funktionalen Hintergrund wurden hingegen ausschließlich positive Effekte in Form von effektiveren Informationsverarbeitungsprozessen verbunden ${ }^{107}$. Der nicht signifikante Gesamtzusammenhang in Bezug auf Gender Diversity in Führungsteams im vorliegenden Beitrag kann als Hinweis darauf gewertet werden, dass beziehungsorientierte Diversity-Merkmale nicht zwangsläufig zu negativen Effekten führen. In dieser Hinsicht korrespondiert der vorliegende Befund mit den Ergebnissen einer jüngst durchgeführten Metaanalyse von Van Dijk/Van Engen/Van Knippenberg (2012) ${ }^{108}$. Die Autoren fanden Hinweise darauf, dass die negativen Effekte von beziehungsorientierter Diversity durch eine systematische Wahrnehmungsverzerrung bei der subjektiven Performanzmessung (z.B. durch Befragung von Führungskräften) bedingt sind. Wird Performanz hingegen mittels objektiver Kennzahlen erhoben, kann ein durchgängig negativer Effekt für beziehungsorientierte Diversity nicht mehr bestätigt werden. Der in der vorliegenden Analyse nachgewiesene nicht signifikante Zusammenhang zwischen Gender Diversity und der mittels objektiver (finanzieller und innovationsbezogener) Kennzahlen gemessenen Unternehmensperformanz insgesamt stützt diese Argumentation.

Mit Blick auf potentielle Randbedingungen des Zusammenhangs zwischen Gender Diversity in Führungsteams und Unternehmensperformanz konnte keiner der untersuchten Moderatoren bestätigt werden. Zwar trifft dies auch für die im Rahmen der Moderationsanalyse vorgenommene Unterscheidung zwischen finanziellen Erfolgskriterien und Innovativität zu, jedoch wurde im Zuge dessen ein zwar sehr schwacher, jedoch signifikant positiver Zusammenhang zwischen Gender Diversity in Führungsteams und finanziellen Erfolgskriterien identifiziert $(\rho=0,027)$. Dieser Zusammenhang weist aber eine beträchtliche Heterogenität in den Effektstärken der Einzelstudien auf und kann deshalb nicht generalisiert werden, was die Aussagekraft des Befundes stark einschränkt. Um die Heterogenität für diesen schwach positiven Zusammenhang aufzulösen und damit die Generalisierbarkeit des positiven Zusammenhangs zumindest für Teams einer bestimmten Größe, einer bestimmten Branche und/oder eines bestimmten Kulturkreises zu er- 
möglichen (vgl. Kapitel 2.2.1-2.2.3), wurden post-hoc erneut Moderationsanalysen für den spezifischen Zusammenhang zwischen Gender Diversity und finanziellen Erfolgskriterien durchgeführt. Auch dabei stellte sich allerdings keiner der untersuchten Moderatoren als signifikant heraus, was abermals gegen eine Generalisierbarkeit des Effekts spricht.

Weitere Moderatoren, die aufgrund der geringen Zahl entsprechender Effektstärken nicht in der vorliegenden Analyse berücksichtigt werden konnten, könnten eventuell zukünftig zur Auflösung der Variabilität in den Ergebnissen der Einzelstudien beitragen (siehe auch Kapitel 5.2). In diesem Zusammenhang könnte etwa die Qualifikation der weiblichen Führungskräfte einen Einfluss darauf besitzen, ob sich Gender Diversity in Führungsteams positiv oder negativ auf die Unternehmensperformanz auswirkt. So finden Smith/Smith/Verner (2006) in ihrer Studie von 2.500 dänischen Unternehmen Hinweise darauf, dass insbesondere dann ein positiver Effekt von Gender Diversity zu erwarten ist, wenn die Frauen im Führungsgremium einen Hochschulabschluss besitzen ${ }^{109}$.

Allgemein sind die Zusammenhänge in der vorliegenden Analyse sehr schwach ausgeprägt und keine der untersuchten Randbedingungen konnte bestätigt werden; daher stellt sich - neben der Suche nach weiteren Moderatoren - generell die Frage nach alternativen Erklärungen, die für diese Befundlage verantwortlich sein könnten. Hierzu sollen im Folgenden drei mögliche Ansätze erörtert werden. Erstens könnte der gegenwärtige Frauenanteil und damit die Gender Diversity in den untersuchten Führungsgremien noch zu gering ausfallen, um die prognostizierten Wirkungen (in Form von sozialen Kategorisierungs- und Informationsverarbeitungsprozessen) zu entfalten. So liegt der Frauenanteil in Führungsteams in den Einzelstudien bei durchschnittlich $10 \%$ und variiert auch innerhalb der Studien nur begrenzt. Dies sollte zum einen Konsequenzen für die angenommenen sozialen Kategorisierungsprozesse in gemischtgeschlechtlichen Führungsteams besitzen. So vermuten etwa Van Knippenberg et al. (2010), dass bei vereinzelten Frauen in Führungsteams die weibliche Minderheit von der männlichen Mehrheit als wenig „bedrohlich“ wahrgenommen wird ${ }^{110}$. Dadurch wird mit den wenigen Frauen auf individueller Ebene und nicht auf Basis sozialer Kategorien interagiert. Soziale Kategorisierungsprozesse und Subgruppenbildung sind entsprechend weniger wahrscheinlich. Zum anderen ist der Group Information Sampling-Theorie zufolge fraglich, ob die spezifischen Sichtweisen der wenigen Frauen in Führungsteams überhaupt diskutiert und bei der Entscheidungsfindung berücksichtigt werden ${ }^{111}$. So zeigen Studien, dass bei Wissensunterschieden zwischen Teammitgliedern verstärkt diejenigen Informationen im Team geteilt und diskutiert werden, über die eine größere Zahl von Teammitgliedern verfügt ${ }^{112}$. Ausschlaggebend hierfür ist unter anderem, dass dadurch die Wahrscheinlichkeit steigt, dass eines der Teammitglieder diese Informationen in die Diskussion einbringt ${ }^{113}$. Bei einer geringen Gender Diversity in Führungsteams ist somit das geschlechterspezifische

109 Vgl. Smith/Smith/Verner (2006).

110 Vgl. Van Knippenberg et al. (2010), S. 328.

111 Vgl. Stasser (1988); Stasser/Titus (1985).

112 Vgl. Brodbeck et al. (2007), S. 464.

113 Vgl. Tindale/Talbot/Martinez (2013), S. 173. 
Wissen von Frauen lediglich einer geringen Zahl von (weiblichen) Teammitgliedern bekannt; dadurch sinkt die Wahrscheinlichkeit, dass diese geschlechterspezifischen Informationen ins Team eingebracht werden.

Ein zweiter Erklärungsansatz könnte in spezifischen Charakteristika von Führungsteams liegen, die die Relevanz von Gender Diversity für eine effektive Zusammenarbeit im Team reduzieren. Es liegt zum Beispiel nahe, dass sich die Mitglieder von Führungsteams durch einen hohen „Need for Cognition“ auszeichnen ${ }^{114}$. Das heißt durch die Art ihrer Tätigkeit sollten die Teammitglieder ohnehin eine hohe Motivation zur Auseinandersetzung mit einer umfassenden und vielfältigen Menge an Informationen besitzen. Entsprechend könnte die geschlechtliche Zusammensetzung für Informationsverarbeitungsprozesse in Führungsteams von untergeordneter Relevanz sein. Darüber hinaus weisen Führungsteams in der Regel eine starke Cross-Funktionalität in ihrer Zusammensetzung auf, da die Teammitglieder einen Großteil ihrer beruflichen Laufbahn in unterschiedlichen Funktionsbereichen verbracht haben ${ }^{115}$. Als Folge daraus könnte das Merkmal Geschlecht durch Unterschiede im funktionalen Hintergrund überlagert werden ${ }^{116}$ : Zum einen könnten soziale Kategorisierungsprozesse auf Basis des Geschlechts weniger wahrscheinlich sein als auf Basis des funktionalen Hintergrunds. Zum anderen sollte auch Cross-Funktionalität mit einer Vielfalt an Wissens- und Erfahrungshintergründen einhergehen, die - im Vergleich zu Gender Diversity - möglicherweise einen stärkeren Einfluss auf die Informationsverarbeitung in Führungsteams besitzen. Schließlich dürften sich die Mitglieder von Führungsteams durch ein hohes Maß an Professionalität auszeichnen $^{117}$. Im Sinne einer möglichst hohen Ziel- beziehungsweise Ergebnisorientierung kann folglich angenommen werden, dass persönliche Merkmale wie das Geschlecht der Teammitglieder geringe Relevanz für eine effiziente Zusammenarbeit besitzen.

Drittens könnte die implizite Gleichsetzung von Gender Diversity mit fähigkeits- und wissensbezogenen Unterschieden von Teammitgliedern unterschiedlichen Geschlechts unzutreffend sein. Wie in der Gender Diversity-Forschung üblich, wurde auch in diesem Beitrag davon ausgegangen, dass Gender Diversity mit Unterschieden hinsichtlich Fähigkeiten und Wissen einhergeht (siehe Kapitel 2.1). Inwiefern jedoch das Geschlecht als sogenanntes „Surface-Level“ Diversity-Merkmal tatsächlich eine angemessene ProxyVariable für (tieferliegende) Fähigkeits- und Wissensunterschiede (sogenannte „Deep-Level“ Diversity) darstellt, konnte in der vorliegenden Analyse nicht geklärt werden. Auch in der bisherigen Diversity-Forschung wurde diese Verbindung kaum empirisch überprüft $^{118}$. Speziell in Bezug auf Führungsteams kann jedoch vermutet werden, dass Gender Diversity, wenn überhaupt, nur schwach mit Deep-Level-Unterschieden verknüpft ist; hierfür sprechen insbesondere zwei Gründe: Zum einen fühlen sich Personen eher zu Gruppen hingezogen, von deren Mitgliedern sie ähnliche Denk- und Verhaltensweisen

114 Vgl. Kearney/Gebert/Voelpel (2009).

115 Vgl. Bunderson/Sutcliffe (2002), S. 881.

116 Vgl. Hogg/Abrams (1988), S. 25.

117 Vgl. Finkelstein/Hambrick/Cannella (2009).

118 Vgl. Van Knippenberg/Schippers (2007), S. 521f. 
erwarten wie ihre eigenen (Attraction-Selection-Attrition-Modell ${ }^{119}$ ). Dies könnte dazu führen, dass sich hauptsächlich Frauen mit typischerweise als maskulin konnotierten Denk- und Verhaltensweisen in die bislang männlich dominierten Führungsteams selektieren; signifikante Deep-Level-Unterschiede zwischen Teammitgliedern unterschiedlichen Geschlechts wären in diesem Fall kaum zu erwarten. Zum anderen könnten solche Selektionseffekte durch mögliche Sozialisationseffekte komplementiert werden. So führen Eagly/Johnson (1990) aus, dass Führungsrollen meist mit klaren Vorstellungen zu angemessenen Verhaltensweisen verknüpft sind ${ }^{120}$. Das Wissen über diese erwarteten (in der Regel maskulin geprägten ${ }^{121}$ ) Verhaltensweisen führt wiederum zu einer Übernahme des geforderten Verhaltens, sodass sich eventuell vorhandene Deep-Level-Unterschiede zwischen weiblichen und männlichen Führungskräften mit der Zeit aufheben könnten.

\subsection{Limitationen und Implikationen für die zukünftige Forschung}

Die vorliegende Analyse weist verschiedene Limitationen auf, die im Folgenden erörtert werden; darauf aufbauend werden Implikationen für die zukünftige Forschung abgeleitet.

Erstens konnte nur eine begrenzte Zahl von Randbedingungen des Zusammenhangs zwischen Gender Diversity in Führungsteams und Unternehmenserfolg untersucht werden. Ausschlaggebend hierfür war die eingeschränkte Datenlage in den Einzelstudien. So konnte etwa der moderierende Einfluss der Dauer der Zusammenarbeit im Team, der Ergebnisinterdependenz oder des Ausmaßes an Teamidentifikation nicht überprüft werden. Diese Randbedingungen wurden bislang nicht für den hier interessierenden Zusammenhang untersucht, erwiesen sich jedoch im Zusammenhang mit anderen DiversityMerkmalen oder bei Nicht-Führungsteams als relevant ${ }^{122}$. Zukünftige Studien zu Gender Diversity in Führungsteams könnten daher möglicherweise ebenfalls von einer Untersuchung dieser Moderatoren profitieren. Die Überprüfung zusätzlicher Randbedingungen ist zudem vor dem Hintergrund wünschenswert, dass die Moderationsanalysen im vorliegenden Beitrag zum Teil auf einer nur geringen Anzahl von Effektstärken basieren. Dies hat zur Folge, dass die Schätzung der durchschnittlichen Zusammenhänge weniger präzise ausfällt. Nach Schmidt et al. (1985) sollte dieser Punkt jedoch nicht überbewertet werden; die Autoren betonen, dass selbst Analysen mit wenigen Effektstärken relevante Erkenntnisse über einzelne Zusammenhänge liefern können ${ }^{123}$.

Zweitens konnten die im Theorieteil beschriebenen vermittelnden Prozesse zwischen Gender Diversity in Führungsteams und Unternehmensperformanz aufgrund der Datenlage in den Einzelstudien nicht empirisch untersucht werden. So zeigte sich zwar in den

119 Vgl. Schneider (1987); Schneider/Goldstein/Smith (1995).

$120 \mathrm{Vgl}$. Eagly/Johnson (1990), S. 234.

121 Vgl. Ryan/Haslam (2007), S. 550f.

122 Vgl. Boerner/Linkohr/Kiefer (2011); Harrison/Price/Bell (1998); Schippers et al. (2003); Van der Vegt/Bunderson (2005).

123 Vgl. Schmidt et al. (1985), S. 749; siehe auch Hülsheger/Anderson/Salgado (2009), S. 1140. 
vergangenen Jahren ein verstärkter Trend zur Untersuchung vermittelnder Prozesse (so genannter „Mediatoren “124) zwischen Diversity und Teamergebnissen ${ }^{125}$, jedoch scheint sich diese Entwicklung trotz wiederholter entsprechender Forderungen bislang nicht im Kontext von Führungsteams durchgesetzt zu haben ${ }^{126}$. Der Fokus auf mögliche Mediatoren wäre jedoch insbesondere aus zwei Gründen auch für Führungsteams von Bedeutung. Zum einen würde das Wissen über vermittelnde Prozesse Erkenntnisse darüber liefern, inwiefern die etablierten Theorien der Diversity-Forschung (Perspektive der Informationsverarbeitung und der sozialen Kategorisierung) auf die Ebene von Führungsteams beziehungsweise den Zusammenhang mit Unternehmensperformanz übertragbar sind. Zum anderen ist durch die Identifikation von Mediatoren eine präzisere Analyse der moderierenden Variablen des Zusammenhangs möglich ${ }^{127}$, zum Beispiel in Form konditional-indirekter Effekte ${ }^{128}$.

Drittens stellt die begrenzte Varianz der Gender Diversity in den Einzelstudien eine Limitation dar. So wurde in kaum einem der untersuchten Führungsteams das maximal mögliche Ausmaß an Gender Diversity (also eine paritätische Besetzung durch Männer und Frauen) erreicht. Die auf Basis dieser Metaanalyse gewonnenen Erkenntnisse beziehen sich demnach hauptsächlich auf den Effekt einer gering bis mäßig stark ausgeprägten Gender Diversity. Daher ist keine Aussage darüber möglich, wie sich der Zusammenhang mit Unternehmensperformanz unter der Bedingung einer sehr hoch ausgeprägten Gender Diversity verändert ${ }^{129}$. Zukünftige Forschung sollte deshalb versuchen, die gesamte Bandbreite an möglicher Gender Diversity in Führungsteams zu berücksichtigen. Dies könnte beispielsweise durch eine verstärkte Berücksichtigung von mittelständischen Unternehmen in Deutschland oder Unternehmen in Nordeuropa gelingen, wo bereits deutlich mehr Frauen in Führungspositionen vertreten sind als im europäischen Durchschnitt ${ }^{130}$.

Viertens ist auf die fehlende Überprüfung der Verbindung zwischen Gender Diversity und tieferliegenden Wissens- und Fähigkeitsunterschieden der gemischtgeschlechtlichen Teammitglieder hinzuweisen. Ein solcher Zusammenhang war zwar in der theoretischen Argumentation implizit angenommen worden, konnte jedoch aufgrund der mangelnden Verfügbarkeit entsprechender Daten in den Einzelstudien nicht empirisch analysiert werden. In der generellen Vernachlässigung des Zusammenhangs zwischen Surface- und Deep-Level-Diversity sehen verschiedene Autoren einen Hauptgrund für das bislang begrenzte Verständnis zur Wirkung von demographischen Diversity-Merkmalen wie dem Geschlecht ${ }^{131}$. Eine gezielte Analyse der Frage, ob und wie Geschlechterunterschiede zu

$124 \mathrm{Vgl}$. Baron/Kenny (1986), S. 1176.

125 Vgl. Phillips et al. (2014), S. 157f.; Van Dijk/Van Engen/Van Knippenberg (2012), S. 49.

126 Vgl. z.B. Boerner/Keding/Hüttermann (2012), S. 59; Forbes/Milliken (1999), S. 502.

127 Vgl. Van Knippenberg/Schippers (2007), S. 533.

128 Vgl. Preacher/Rucker/Hayes (2007)

129 Vgl. Van Knippenberg et al. (2010), S. 328f.

130 Vgl. Commerzbank AG (2011); Europäische Kommission (2014).

131 Vgl. Certo et al. (2006), S. 834; Jackson/Joshi (2011), S. 668f.; Van Knippenberg/Schippers (2007), S. 521f. 
einer Vielfalt an Wissen, Erfahrungen und Perspektiven in einem Team führen, stellt daher ein relevantes und innovatives Forschungsfeld dar. In diesem Zusammenhang erscheint auch eine Untersuchung der im vorangegangenen Kapitel diskutierten möglichen Selektions- und Sozialisationseffekte von männlichen und weiblichen Führungskräften als sinnvoll. Im Rahmen von Längsschnittanalysen könnte dabei die Angleichung möglicher Deep-Level-Unterschiede zwischen männlichen und weiblichen Führungskräften über die Zeit analysiert werden.

Fünftens wurde der Zusammenhang sogenannter „Faultlines“ in Führungsteams mit Unternehmensperformanz in dieser Metaanalyse nicht berücksichtigt. Faultlines beschreiben hypothetische Trennlinien in einem Team, die durch eine systematische Verteilung mehrerer Diversity-Merkmale entstehen ${ }^{132}$. Starke Faultlines existieren, wenn unterschiedliche Diversity-Merkmale hoch miteinander korreliert sind (z.B. alle älteren Teammitglieder sind männlich und alle jüngeren weiblich). Ihnen wird ein noch stärkerer Effekt auf die Entstehung von Subgruppen in Teams zugeschrieben ${ }^{133}$. Gegen die Berücksichtigung derartiger Faultlines im vorliegenden Beitrag sprach aber, dass diese den Rückschluss auf die spezifische Wirkung des Merkmals Geschlecht erschweren. Zudem ergab die Literaturrecherche, dass Faultlines bis auf einzelne Ausnahmen bislang nicht für Führungsteams untersucht wurden ${ }^{134}$. Die Faultline-Forschung für Nicht-Führungsteams deutet jedoch darauf hin, dass bei der Kombination von verschiedenen Diversity-Merkmalen signifikante Effekte zu erwarten sind und mehr Varianz erklärt werden kann als bei der Fokussierung auf ein einzelnes Merkmal ${ }^{135}$. Entsprechend könnte eine Untersuchung von Faultlines in Verbindung mit Gender Diversity in Führungsteams möglicherweise weiteren Aufschluss über den Zusammenhang mit Unternehmensperformanz geben.

\subsection{Implikationen für die Praxis}

Die Ableitung praktischer Implikationen für Unternehmen aus der vorliegenden Analyse ist insbesondere aus zwei Gründen nur unter Vorbehalt möglich: Zum einen untersuchen die in der Metaanalyse eingeschlossenen Studien ausschließlich nicht-deutsche Unternehmen. Entsprechend ist die Übertragbarkeit der Ergebnisse des vorliegenden Beitrags auf den deutschen Kontext nicht ohne Weiteres möglich. Zum anderen muss bei der Ableitung von Implikationen für die Unternehmenspraxis der Gefahr eines ökologischen Fehlschlusses Rechnung getragen werden ${ }^{136}$. So kann beispielsweise auf Basis der Ergebnisse dieser Metaanalyse lediglich die Schlussfolgerung gezogen werden, dass die Unternehmensperformanz insgesamt offenbar unabhängig vom Ausmaß an Gender Diversity in den Einzelstudien ist. Obgleich dies - unter Vermeidung eines ökologischen Fehlschlusses - nicht für jedes einzelne Unternehmen zutreffen muss, besitzt der Befund 
zumindest in der Tendenz eine gewisse Aussagekraft hinsichtlich der Effekte von Gender Diversity in Führungsgremien.

Erstens deuten der nicht signifikante Zusammenhang mit Unternehmensperformanz insgesamt sowie der nicht generalisierbare Effekt auf finanzielle Erfolgskennzahlen darauf hin, dass Gender Diversity nicht gleichsam „automatisch“ zu positiven ökonomischen Effekten führt. Bezugnehmend auf die zu Beginn dieses Beitrags angesprochene öffentliche Debatte scheint daher Vorsicht geboten, etwa die Einführung von Frauenquoten in Führungsgremien primär über mögliche ökonomische Vorteile zu begründen. Hierfür wäre zunächst noch mehr Forschung notwendig, um beispielsweise entsprechende Randbedingungen zu identifizieren, unter denen sich mögliche ökonomische Vorteile von Gender Diversity tatsächlich realisieren. Nach derzeitigem Stand könnte jedoch eine ausschließlich ökonomische Legitimation sogar schädlich sein. Denn falls sich die diesbezüglich erhofften positiven Effekte nicht unmittelbar einstellen sollten, könnte dies zu einer Diskreditierung des Gender Diversity-Gedankens insgesamt führen.

Zweitens besitzt der nicht generalisierbare Zusammenhang zwischen Gender Diversity in Führungsgremien und Unternehmensperformanz eine weitere wichtige Implikation: So lassen sich auch „Unkenrufe“ nicht bestätigen, die in der Erhöhung von Gender Diversity in Führungsteams zwangsläufig eine Schwächung der Unternehmensleistung sehen. Daraus folgt zudem, dass beim Thema Gender Diversity in Führungsgremien gesellschaftlich-normative Forderungen nach einer Erhöhung der Frauenquote („moral justice case“) nicht unbedingt im Widerspruch zu wirtschaftlichen Überlegungen („business case“) stehen müssen. Vielmehr deuten die Ergebnisse der vorliegenden Analyse darauf hin, dass eine Erhöhung der Gender Diversity im Sinne des „moral justice case“ durchaus erfolgen kann, ohne dass dies notwendigerweise negative Konsequenzen für den „business case“ hätte.

Schließlich erscheint es denkbar, dass sich im Zuge einer normativ legitimierten Erhöhung von Gender Diversity in Führungsgremien möglicherweise in Zukunft ökonomische Vorteile ergeben. So könnte die aktive Frauenförderung die Reputation der jeweiligen Unternehmen insbesondere bei Arbeitnehmerinnen steigern und dadurch die Chancen des Unternehmens auf dem Rekrutierungsmarkt verbessern ${ }^{137}$. Derartige Rekrutierungsvorteile könnten angesichts steigender Qualifikationsniveaus weiblicher Bewerberinnen $^{138}$ und des sich verschärfenden Fachkräftemangels ${ }^{139}$ in den kommenden Jahren weiter an Bedeutung gewinnen. 


\section{Literaturverzeichnis}

Die mit einem Asterisk gekennzeichneten Quellen fanden Berücksichtigung in der Metaanalyse.

*Abdullah, Shamsul N./Ismail, Ku N. I./Nachum, Lilach (2012), Women on Boards of Malaysian Firms: Impact on Market and Accounting Performance, Arbeitspapier.

Ali, Muhammad/Kulik, Carol T./Metz, Isabel (2011), The Gender Diversity-Performance Relationship in Services and Manufacturing Organizations, in: International Journal of Human Resource Management, Vol. 22, S. 1464-1485.

Allen, Tammy D./Eby, Lillian T./Poteet, Mark L./Lentz, Elizabeth/Lima, Lizzette (2004), Career Benefits Associated With Mentoring for Proteges: A Meta-Analysis, in: Journal of Applied Psychology, Vol. 89, S. 127-136.

Amason, Allen C. (1996), Distinguishing the Effects of Functional and Dysfunctional Conflict on Strategic Decision Making: Resolving a Paradox for Top Management Teams, in: Academy of Management Journal, Vol. 39, S. $123-148$.

Amason, Allen C./Sapienza, Harry J. (1997), The Effects of Top Management Team Size and Interaction Norms on Cognitive and Affective Conflict, in: Journal of Management, Vol. 23, S. 495-516.

Ashforth, Blake E./Johnson, Scott A. (2001), Which Hat to Wear? The Relative Salience of Multiple Identities in Organizational Contexts, in: Hogg, Michael A./Terry, Deborah J. (Hrsg.), Social Identity Processes in Organizational Contexts, Philadelphia, S. 31-48.

Baer, Markus/Frese, Michael (2003), Innovation Is Not Enough: Climates for Initiative and Psychological Safety, Process Innovations, and Firm Performance, in: Journal of Organizational Behavior, Vol. 24, S. 45-68.

Balkin, David B./Markman, Gideon D./Gomez-Mejia, Luis R. (2000), Is CEO Pay in High-technology Firms Related to Innovation?, in: Academy of Management Journal, Vol. 43, S. 1118-1129.

Bantel, Karen A.JJackson, Susan E. (1989), Top Management and Innovations in Banking: Does the Composition of the Top Team Make a Difference?, in: Strategic Management Journal, Vol. 10, S. 107-124.

Baron, Reuben M./Kenny, David A. (1986), The Moderator-Mediator Variable Distinction in Social Psychological Research: Conceptual, Strategic, and Statistical Considerations, in: Journal of Personality and Social Psychology, Vol. 51, S. 1173.

*Bear, Stephen/Rahman, Noushi/Post, Corinne (2010), The Impact of Board Diversity and Gender Composition on Corporate Social Responsibility and Firm Reputation, in: Journal of Business Ethics, Vol. 97, S. 207-221.

Bell, Suzanne T./Villado, Anton J./Lukasik, Marc A./Belau, Larisa/Briggs, Andrea L. (2011), Getting Specific about Demographic Diversity Variable and Team Performance Relationships: A Meta-Analysis, in: Journal of Management, Vol. 37, S. 709-743.

Blau, Peter M. (1977), Inequality and heterogeneity, 1. Aufl., New York.

Boerner, Sabine/Keding, Hannah/Hüttermann, Hendrik (2012), Gender Diversity und Organisationserfolg: Eine kritische Bestandsaufnahme, in: zfbf, 64. Jg., S. 37-70.

Boerner, Sabine/Linkohr, Marius/Kiefer, Sabine (2011), Top Management Team Diversity: Positive in the Short Run, but Negative in the Long Run?, in: Team Performance Management, Vol. 17, S. 328-353.

*Boulouta, Ioanna (2013), Hidden Connections: The Link Between Board Gender Diversity and Corporate Social Performance, in: Journal of Business Ethics, Vol. 113, S. 185-197.

Bowen, David E./Schneider, Benjamin (1988), Services Marketing and Management: Implications for Organizational Behavior, in: Staw, Barry M./Cummings, Larry L. (Hrsg.), Research in Organizational Behavior, Greenwich, CT, S. 43-80.

Bowers, Clint A./Pharmer, James A./Salas, Eduardo (2000), When Member Homogeneity is Needed in Work Teams: A Meta-Analysis, in: Small Group Research, Vol. 31, S. 305-327. 
*Brammer, Stephen/Millington, Andrew/Pavelin, Stephen (2009), Corporate Reputation and Women on the Board, in: British Journal of Management, Vol. 20, S. 17-29.

Brodbeck, Felix C./Kerschreiter, RudolflMojzisch, Andreas/Schulz-Hardt, Stefan (2007), Group Decision Making under Conditions of Distributed Knowledge: The Information Asymmetries Model, in: Academy of Management Review, Vol. 32, S. 459-479.

Bunderson, J. Stuart/Sutcliffe, Kathleen M. (2002), Comparing Alternative Conceptualizations of Functional Diversity in Management Teams: Process and Performance Effects, in: Academy of Management Journal, Vol. 45, S. 875-893.

Bundesagentur für Arbeit (2012), Der Arbeitsmarkt in Deutschland: Frauen und Männer am Arbeitsmarkt im Jahr 2012, http://statistik.arbeitsagentur.de/Statischer-Content/Arbeitsmarktberichte/Personengruppen/generischePublikationen/Frauen-Maenner-Arbeitsmarkt-2013-07.pdf (26.03.2014).

Byrne, Donn E. (1971), The Attraction Paradigm, 1. Aufl., New York.

Byrnes, James P./Miller, David C./Schafer, William D. (1999), Gender Differences in Risk Taking: A Meta-Analysis, in: Psychological Bulletin, Vol. 125, S. 367.

Cabrera, Susan F./Sauer, Stephen J./Thomas-Hunt, Melissa C. (2009), The Evolving Manager Stereotype: The Effects of Industry Gender Typing on Performance Expectations for Leaders and Their Teams, in: Psychology of Women Quarterly, Vol. 33, S. 419-428.

${ }^{*}$ Cannella, Alberta A./Park, Jong H./Lee, Ho U. (2008), Top Management Team Functional Background Diversity and Firm Performance: Examining the Roles of Team Member Colocation and Environmental Uncertainty, in: Academy of Management Journal, Vol. 51, S. 768-784.

Certo, S. Trevis/Lester, Richard H./Dalton, Catherine M./Dalton, Dan R. (2006), Top Management Teams, Strategy and Financial Performance: A Meta-Analytic Examination, in: Journal of Management Studies, Vol. 43, S. 813-839.

Chakrabarti, Alok K. (1974), The Role of Champion in Product Innovation, in: California Management Review, Vol. 17, S. 58-62.

Chatman, Jennifer A./Polzer, Jeffrey T./Barsade, Sigal G./Neale, Margaret A. (1998), Being Different Yet Feeling Similar: The Influence of Demographic Composition and Organizational Culture on Work Processes and Outcomes, in: Administrative Science Quarterly, Vol. 43, S. 749-780.

Chatman, Jennifer A./Spataro, Sandra E. (2005), Using Self-Categorization Theory to Understand Relational Demography-Based Variations in People's Responsiveness to Organizational Culture, in: Academy of Management Journal, Vol. 48, S. 321-331.

Commerzbank AG (2011), Aktuelle Commerzbank-Studie: Der Mittelstand führt: 20 Prozent der Topführungskräfte sind Frauen, https:/www.commerzbank.de/de/hauptnavigation/presse/pressemitteilungen/archiv1/2011/quartal_11_02/presse_archiv_detail_11_02_8535.html (05.03.2015).

Cramton, Catherine D./Hinds, Pamela J. (2005), Subgroup Dynamics in Internationally Distributed Teams: Ethnocentrism or Cross-National Learning?, in: Staw, Barry M./Kramer, Roderick M. (Hrsg.), Research in Organizational Behavior. An Annual Series of Analytical Essays and Critical Reviews, Oxford, S. 231-263.

Curral, Luis A./Forrester, Rosalind H./Dawson, Jeremy F./West, Michael A. (2001), It's What You Do and the Way that You Do it: Team Task, Team Size, and Innovation-Related Group Processes, in: European Journal of Work and Organizational Psychology, Vol. 10, S. 187-204.

*Darmadi, Salim (2011), Board Diversity and Firm Performance: The Indonesian Evidence, in: Corporate Ownership and Control, Vol. 9, S. 524-539.

De Wit, Frank R. C./Greer, Lindred L./Jehn, Karen A. (2012), The Paradox of Intragroup Conflict: A Meta-Analysis, in: Journal of Applied Psychology, Vol. 97, S. 360. 
Deutscher Bundestag (2011), Bundestag lehnt eine gesetzliche Frauenquote ab, https://www.bundestag.de/dokumente/textarchiv/2011/36878087_kw48_de_frauenquote/207116 (23.06.2014).

Deutscher Bundestag (2015), Frauenquote für Top-Positionen beschlossen, http://www.bundestag.de/dokumente/ textarchiv/2015/kw10_de_frauenquote/363058 (09.03.2015)

*Dezsö, Cristian L./Ross, David G. (2012), Does Female Representation in Top Management Improve Firm Performance? A Panel Data Investigation, in: Strategic Management Journal, Vol. 33, S. 1072-1089.

Eagly, Alice H./Johnson, Blair T. (1990), Gender and Leadership Style: A Meta-Analysis, in: Psychological Bulletin, Vol. 108, S. 233-256.

Elenkov, Detelin S./Manev, Ivan M. (2005), Top Management Leadership and Influence on Innovation: The Role of Sociocultural Context, in: Journal of Management, Vol. 31, S. 381-402.

Elmerich, Kathrin/Hornberger, Sonia/Watrinet, Christine (2007), Inhalts- und Wirkungsanalyse von Leitbildern unter dem Aspekt Managing Diversity, in: Wagner, Dieter/Voigt, Bernd-Friedrich (Hrsg.), Diversity-Management als Leitbild von Personalpolitik, Wiesbaden, S. 17-38.

Emrich, Cynthia G./Denmark, Florence L./Den Hartog, Deanne N. (2004), Cross-Cultural Differences in Gender Egalitarianism, in: House, Robert J./Hanges, Paul J./Javidan, Mansour/Dorfman, Peter W./Gupta, Vipin (Hrsg.), Culture, Leadership, and Organizations, Thousand Oaks, S. 343-394.

Engelen, Andreas/Brettel, Malte (2011), Assessing Cross-Cultural Marketing Theory and Research, in: Journal of Business Research, Vol. 64, S. 516-523.

Europäische Kommission (2014), Gender balance in decision-making positions, http://ec.europa.eu/justice/gender-equality/gender-decision-making/database/business-finance/executives-non-executives/index_en.htm (05.03.2015).

Fargues, Philippe/McCormick, Ashley (2013), Ageing of skills and complementary immigration in the EU, 2010-2025, in: European University Institute Working Papers, Vol. RSCAS 2013/81.

Feingold, Alan (1994), Gender Differences in Personality: A Meta-Analysis, in: Psychological Bulletin, Vol. 116, S. $429-456$.

Finkelstein, Sydney/Hambrick, Donald C./Cannella, Albert A. (2009), Strategic Leadership: Theory and Research on Executives, Top Management Teams, and Boards, 1. Auflage, New York.

Finkelstein, Sydney/Mooney, Ann C. (2003), Not the Usual Suspects: How to Use Board Process to Make Boards Better, in: Academy of Management Executive, Vol. 17, S. 101-113.

Fiske, Susan T. (1993), Controlling other People: The Impact of Power on Stereotyping, in: American Psychologist, Vol. 48, S. 621-628.

Fiske, Susan T./Lin, Monica/Neuberg, Steven L. (1999), The Continuum Model: Ten Years Later, in: Chaiken, Shelly/ Trope, Yaacov (Hrsg.), Dual-Process Theories in Social Psychology, New York, S. 231-254.

Forbes, Daniel P./Milliken, Frances J. (1999), Cognition and Corporate Governance: Understanding Boards of Directors as Strategic Decision-Making Groups, in: Academy of Management Review, Vol. 24, S. 489-505.

*Galbreath, Jeremy (2011), Are there Gender-Related Influences on Corporate Sustainability? A Study of Women on Boards of Directors, in: Journal of Management \& Organization, Vol. 17, S. 17-38.

*Gallego-Alvarez, Isabel/García-Sánchez, Isabel M./Rodríguez-Dominguez, Luis (2010), The Influence of Gender Diversity on Corporate Performance, in: Revista de Contabilidad-Spanish Accounting Review, Vol. 13, S. 53-88.

Gibson, Cristina B. (2001), From Knowledge Accumulation to Accommodation: Cycles of Collective Cognition in Work Groups, in: Journal of Organizational Behavior, Vol. 22, S. 121-134.

Gooding, Richard Z./Wagner, John A. (1985), A Meta-Analytic Review of the Relationship between Size and Performance: The Productivity and Efficiency of Organizations and Their Subunits, in: Administrative Science Quarterly, Vol. 30, S. 462-481. 
Grönroos, Christian (2006), Adopting a Service Logic for Marketing, in: Marketing Theory, Vol. 6, S. $317-333$.

Guzzo, Richard A./Shea, Gregory P. (1992), Group Performance and Intergroup Relations in Organizations, in: Dunnette, Marvin D./Hough, Leaetta M. (Hrsg.), Handbook of Industrial and Organizational Psychology, Palo Alto, S. 269-313.

*Hafsi, Tä̈eb/Turgut, Gokhan (2013), Boardroom Diversity and its Effect on Social Performance: Conceptualization and Empirical Evidence, in: Journal of Business Ethics, Vol. 112, S. 463-479.

Hagedoorn, John/Cloodt, Myriam (2003), Measuring Innovative Performance: Is there an Advantage in Using Multiple Indicators?, in: Research Policy, Vol. 32, S. 1365-1379.

*Hagendorff, Jens/Keasey, Kevin (2010), The Value of Board Diversity in Banking: Evidence from the Market for Corporate Control, in: The European Journal of Finance, Vol. 18, S. 41-58.

Halpern, Diane F. (2004), A Cognitive-Process Taxonomy for Sex Differences in Cognitive Abilities, in: Current Directions in Psychological Science, Vol. 13, S. 135-139.

Hambrick, Donald C./Mason, Phyllis A. (1984), Upper Echelons: The Organization as a Reflection of Its Top Managers, in: Academy of Management Review, Vol. 9, S. 193-206.

Harrison, David A./Price, Kenneth H./Bell, Myrtle P. (1998), Beyond Relational Demography: Time and the Effects of Surface-and Deep-level Diversity on Work Group Cohesion, in: Academy of Management Journal, Vol. 41, S. 96-107.

*Haslam, Alexander S./Ryan, Michelle K./Kulich, Clara/Trojanowski, Grzegorz/Atkins, Cate (2010), Investing with Prejudice: The Relationship Between Women's Presence on Company Boards and Objective and Subjective Measures of Company Performance, in: British Journal of Management, Vol. 21, S. 484-497.

*He, Jinyu/Huang Zhi (2011), Board Informal Hierarchy and Firm Financial Performance: Exploring a Tacit Structure Guiding Boardroom Interactions, in: Academy of Management Journal, Vol. 54, S. 1119-1139.

Hedges, Larry V./Olkin, Ingram (1985), Statistical methods for meta-analysis, Orlando.

Hinsz, Verlin B./Tindale, R. Scott/Vollrath, David A. (1997), The Emerging Conceptualization of Groups as Information Processors, in: Psychological Bulletin, Vol. 121, S. 43-64.

Hirschfeld, Robert R./Jordan, Mark H./Feild, Hubert S./Giles, William F./Armenakis, Achilles A. (2005), Teams' Female Representation and Perceived Potency as Inputs to Team Outcomes in a Predominantly Male Field Setting, in: Personnel Psychology, Vol. 58, S. 893-924.

Hogg, Michael A./Abrams, Dominic (1988), Social Identifications: A Social Psychology of Intergroup Relations and Group Processes, 1. Aufl., London.

Holst, Elke/Busch, Anne/Kröger, Lea (2012), Führungskräfte-Monitor 2012: Update 2001 - 2010, in: DIW Berlin: Politikberatung kompakt, S. 1-145.

Holst, Elke/Kirsch, Anja (2014), Frauen sind in Vorständen großer Unternehmen in Deutschland noch immer die Ausnahme - moderat steigende Anteile in Aufsichtsräten, in: DIW Wochenbericht Nr. 3, S. 19-31.

Holst, Elke/Kirsch, Anja (2015), Weiterhin kaum Frauen in den Vorständen großer Unternehmen - auch Aufsichtsräte bleiben Männerdomänen, in: DIW Wochenbericht Nr. 4, S. 47-60.

Homan, Astrid C./Greer, Lindred L./Jehn, Karen A./Koning, Lukas (2010), Believing Shapes Seeing: The Impact of Diversity Beliefs on the Construal of Group Composition, in: Group Processes \& Intergroup Relations, Vol. 13, S. 477-493.

Homan, Astrid C./Van Knippenberg, Daan/Van Kleef, Gerben A./De Dreu, Carsten K. W. (2007), Bridging Faultlines by Valuing Diversity: Diversity Beliefs, Information Elaboration, and Performance in Diverse Work Groups, in: Journal of Applied Psychology, Vol. 92, S. 1189-1199.

Horwitz, Sujin K./Horwitz, Irwin B. (2007), The Effects of Team Diversity on Team Outcomes: A Meta-Analytic Review of Team Demography, in: Journal of Management, Vol. 33, S. 987-1015. 
House, Robert J./Hanges, Paul J./Javidan, Mansour/Dorfman, Peter W./Gupta, Vipin (2004), Culture, Leadership, and Organizations, 1. Aufl., Thousand Oaks.

House, Robert J./Javidan, Mansour (2004), Overview of GLOBE, in: House, Robert J./Hanges, Paul J./Javidan, Mansour/ Dorfman, Peter W./Gupta, Vipin (Hrsg.), Culture, Leadership, and Organizations, Thousand Oaks, S. 9-28.

Huffcutt, Allen I./Arthur, Winfred (1995), Development of a New Outlier Statistic for Meta-Analytic Data, in: Journal of Applied Psychology, Vol. 80, S. 327-334.

Hülsheger, Ute R./Anderson, Neil/Salgado, Jesus F. (2009), Team-Level Predictors of Innovation at Work: A Comprehensive Meta-Analysis Spanning Three Decades of Research, in: Journal of Applied Psychology, Vol. 94, S. $1128-1145$.

Hunter, John E./Schmidt, Frank L. (2007), Methods of Meta-Analysis, 2. Aufl., Thousand Oaks.

*Ittonen, Kim/Miettinen, Johanna/Vahamaa, Sami (2010), Does Female Representation on Audit Committees Affect Audit Fees?, in: Quarterly Journal of Finance and Accounting, Vol. 49, S. 113-139.

Jackson, Susan E./Joshi, Aparna (2011), Work Team Diversity, in: Zedeck, Sheldon (Hrsg.), APA Handbook of Industrial and Organizational Psychology, Washington D.C, S. 651-686.

Jackson, Susan E./Schuler, Randall S./Rivero, J. Carlos (1989), Organizational Characteristics as Predictors of Personnel Practices, in: Personnel Psychology, Vol. 42, S. 727-786.

Jehn, Karen A./Northcraft, Gregory B./Neale, Margaret A. (1999), Why Differences Make a Difference: A Field Study of Diversity, Conflict, and Performance in Workgroups, in: Administrative Science Quarterly, Vol. 44, S. 741-763. Joshi, Aparna/Roh, Hyuntak (2009), The Role of Context in Work Team Diversity Research: A Meta-Analytic Review, in: Academy of Management Journal, Vol. 52, S. 599-627.

*Julizaerma, M. K./Sori, Zulkarnain M. (2012), Gender Diversity in the Boardroom and Firm Performance of Malaysian Public Listed Companies, in: International Congress on Interdisciplinary Business and Social Sciences 2012, Vol. 65, S. 1077-1085.

*Kang, Eugene/Ding, David K./Charoenwong, Charlie (2010), Investor Reaction to Women Directors, in: Journal of Business Research, Vol. 63, S. 888-894.

Kearney, Eric/Gebert, Diether/Voelpel, Sven C. (2009), When and How Diversity Benefits Teams: The Importance of Team Members' Need for Cognition, in: Academy of Management Journal, Vol. 52, S. 581-598.

Klein, Cameron/DiazGranados, Deborah/Salas, Eduardo/Le, Huy/Burke, Shaun C./Lyons, Rebecca/Goodwin, Gerald F. (2009), Does Team Building Work?, in: Small Group Research, Vol. 40, S. 181-222.

Köppel, Petra (2010), Diversity Management in Deutschland — eine Unternehmensbefragung, in: Badura, Bernhard/ Schröder, Helmut/Klose, Joachim/Macco, Katrin (Hrsg.), Fehlzeiten-Report 2010, S. 23-35.

Krell, Gertraude/Ortlieb, Renate/Sieben, Barbara (2011), Chancengleichheit durch Personalpolitik: Gleichstellung von Frauen und Männern in Unternehmen und Verwaltung, 6. Aufl., Wiesbaden.

*Krishnan, Hema A./Park, Daewoo (2005), A Few Good Women—on Top Management Teams, in: Journal of Business Research, Vol. 58, S. 1712-1720.

Kunze, Florian/Boehm, Stephan A. (2013), Research on Age Diversity in the Workforce: Current Trends and Future Research Directions, in: Field, John/Burke, Ronald J. (Hrsg.), The SAGE handbook of aging, work and society, London, S. 41-59.

Lau, Dora C./Murnighan, J. K. (1998), Demographic Diversity and Faultlines: The Compositional Dynamics of Organizational Groups, in: Academy of Management Review, Vol. 23, S. 325-340.

*Lehobo, Lineo (2011), The Relationship Between Diversity and Corporate Profitability: The Top 100 Companies on the JSE Ltd, Unveröffentlichte Masterarbeit, University of Johannesburg, Johannesburg.

Lindstädt, Hagen/Wolff, Michael/Fehre, Kerstin (2011), Frauen in Führungspositionen: Auswirkungen auf den Unternehmenserfolg, http://www.bmfsfj.de/BMFSFJ/Service/Publikationen/publikationsliste,did=175142.htm (19.06.2014). 
Lipsey, Mark W./Wilson, David B. (2007), Practical Meta-Analysis, 8. Aufl., Thousand Oaks.

*Lückerath-Rovers, Mijntje (2013), Women on Boards and Firm Performance, in: Journal of Management \& Governance, Vol. 17, S. 491-509.

*Mahadeo, JyotiD/Soobaroyen, Teerooven/Hanuman, Vanisha Oogarah (2012), Board Composition and Financial Performance: Uncovering the Effects of Diversity in an Emerging Economy, in: Journal of Business Ethics, Vol. 105, S. 375-388.

*Miller, Toyah/Triana, Maria del Carmen (2009), Demographic Diversity in the Boardroom: Mediators of the Board Diversity-Firm Performance Relationship, in: Journal of Management Studies, Vol. 46, S. 755-786.

Murray, Alan I. (1989), Top Management Group Heterogeneity and Firm Performance, in: Strategic Management Journal, Vol. 10, S. 125-141.

*Olson, Bradley J./Parayitam, Satyanarayana/Twigg, Nicholas W. (2006), Mediating Role of Strategic Choice Between Top Management Team Diversity and Firm Performance: Upper Echelons Theory Revisited, in: Journal of Business \& Management, Vol. 12, S. 111-126.

* Özatac, Nesrin (2011), Gender Diversity in Board of Directors and Top Management: The Case of Turkish Banks, in: Actual Problems of Economics, Vol. 1, S. 311-319.

*Pathan, Shams/Faff, Robert (2013), Does Board Structure in Banks Really Affect Their Performance?, in: Journal of Banking \& Finance, Vol. 37, S. 1573-1589.

Pearsall, Matthew J./Ellis, Aleksander P. J./Evans, Joel M. (2008), Unlocking the Effects of Gender Faultlines on Team Creativity: Is Activation the Key?, in: Journal of Applied Psychology, Vol. 93, S. 225-234.

Preacher, Kristopher J./Rucker, Derek D./Hayes, Andrew F. (2007), Addressing Moderated Mediation Hypotheses: Theory, Methods, and Prescriptions, in: Multivariate Behavioral Research, Vol. 42, S. 185-227.

Phillips, Katherine W./Duguid, Michelle/Thomas-Hunt, Melissa/Upadhyay, Arun (2014), Diversity as Knowledge Exchange: The Roles of Information Processing, Expertise, and Status, in: Roberson, Quinetta M. (Hrsg.), The Oxford handbook of diversity and work, Oxford, S. 157-178.

*Puthenpurackal, John/Upadhyay, Arun (2010), Board Gender Diversity and Firm Performance: The Impact of Information Environment, Arbeitspapier.

*Rambo, Charles M. (2013), Influence of the Capital Markets Authority's Corporate Governance Guidelines on Financial Performance of Commercial Banks in Kenya, in: The International Journal of Business and Finance Research, Vol. 7, S. 77-92.

Reskin, Barbara F./McBrier, Debra B./Kmec, Julie A. (1999), The Determinants and Consequences of Workplace Sex and Race Composition, in: Annual Review of Sociology, Vol. 25, S. 335.

Richard, Orlando C./Murthi, B. P./Ismail, Kiran (2007), The Impact of Racial Diversity on Intermediate and Long Term Performance: The Moderating Role of Environmental Context, in: Strategic Management Journal, Vol. 28, S. 1213-1233.

Robinson, William S. (1950), Ecological Correlations and the Behavior of Individuals, in: American Sociological Review, Vol. 15, S. 351-357.

*Rodriguez-Dominguez, Luis/Garcia-Sanchez, Isabel-Maria/Gallego-Alvarez, Isabel (2012), Explanatory Factors of the Relationship Between Gender Diversity and Corporate Performance, in: European Journal of Law and Economics, Vol. 33, S. 603-620.

*Roh, Hyuntak (2010), Unraveling the Effects of Workforce Diversity on Firm Performance: The Role of HRM Practices in Large U.S. Corporate Law Firms, Unveröffentlichte Dissertation, University of Illinois at UrbanaChampaign, Urbana.

*Rost, Katja/Osterloh, Margit (2010), Opening the Black Box of Upper Echelons: Drivers of Poor Information Processing During the Financial Crisis, in: Corporate Governance: An International Review, Vol. 18, S. $212-233$. 
Ryan, Michelle K./Haslam, Alexander S. (2007), The Glass Cliff: Exploring the Dynamics Surrounding the Appointment of Women to Precarious Leadership Positions, in: The Academy of Management Review, Vol. 32, S. 549-572.

Schippers, Michaéla C./Den Hartog, Deanne N./Koopman, Paul L./Wienk, Janique A. (2003), Diversity and Team Outcomes: The Moderating Effects of Outcome Interdependence and Group Longevity and the Mediating Effect of Reflexivity, in: Journal of Organizational Behavior, Vol. 24, S. 779-802.

Schmidt, Frank L./Hunter, John E./Pearlman, Kenneth/Hirsh, Hannah R. (1985), Forty Questions about Validity Generalization and Meta-Analysis, in: Personnel Psychology, Vol. 38, S. 697-798.

Schneider, Benjamin (1987), The People Make the Place, in: Personnel Psychology, Vol. 40, S. 437-453.

Schneider, Benjamin/Goldstein, Harold W./Smith, D. B. (1995), The ASA Framework: An Update, in: Personnel Psychology, Vol. 48, S. 747-773.

*Schwizer, Paola/Soana, Maria-Gaia/Cucinelli, Doriana (2010), The Relationship between Board Diversity and Firm Performance: The Italian Evidence, Arbeitspapier.

*Shrader, Charles B./Blackburn, Virginia B./Iles, Paul (1997), Women in Management and Firm Financial Performance: An Exploratory Study, in: Journal of Managerial Issues, Vol. 9, S. 355-372.

*Shukeri, Siti N./Shin, Ong W./Shaari, Mohd S. (2012), Does Board of Director's Characteristics Affect Firm Performance? Evidence from Malaysian Public Listed Companies, in: International Business Research, Vol. 5, S. $120-127$.

Simons, Tony/Pelled, Lisa H./Smith, Ken A. (1999), Making Use of Difference: Diversity, Debate, and Decision Comprehensiveness in Top Management Teams, in: Academy of Management Journal, Vol. 42, S. 662-673.

Smith, Nina/Smith, Valdemar/Verner, Mette (2006), Do Women in Top Management Affect Firm Performance? A Panel Study of 2,500 Danish Firms, in: International Journal of Productivity and Performance Management, Vol. 55, S. 569-593.

Stahl, Günter K./Maznevski, Martha L./Voigt, Andreas/Jonsen, Karsten (2010), Unraveling the Effects of Cultural Diversity in Teams: A Meta-Analysis of Research on Multicultural Work Groups, in: Journal of International Business Studies, Vol. 41, S. 690-709.

Stasser, Garold (1988), Computer Simulation as a Research Tool: The DISCUSS Model of Group Decision Making, in: Journal of Experimental Social Psychology, Vol. 24, S. 393-422.

Stasser, Garold/Titus, William (1985), Pooling of Unshared Information in Group Decision Making: Biased Information Sampling During Discussion, in: Journal of Personality and Social Psychology, Vol. 48, S. 1467-1478.

Statistisches Bundesamt (2012), Frauen und Männer auf dem Arbeitsmarkt: Deutschland und Europa, https://www. destatis.de/DE/Publikationen/Thematisch/Arbeitsmarkt/Erwerbstaetige/BroeschuereFrauenMaennerArbeitsmarkt0010018129004.pdf?_blob=publicationFile (04.03.2015).

Statistisches Bundesamt (2014), Arbeitskräfteerhebung: Bevölkerung, Erwerbstätige, Erwerbslose, Erwerbspersonen, Nichterwerbspersonen, https:/www-genesis.destatis.de/genesis/online/link/tabelleErgebnis/12211-0001 (06.06.2014).

Stelter, Nicole Z. (2002), Gender Differences in Leadership: Current Social Issues and Future Organizational Implications, in: Journal of Leadership \& Organizational Studies, Vol. 8, S. 88-99.

Stewart, Greg L. (2006), A Meta-Analytic Review of Relationships Between Team Design Features and Team Performance, in: Journal of Management, Vol. 32, S. 29-55.

Süß, Stefan (2008), Diversity-Management auf dem Vormarsch. Eine empirische Analyse der deutschen Unternehmenspraxis, in: zfbf, 60. Jg., S. 406-430.

Süß, Stefan/Kleiner, Markus (2006), Diversity-Management in Deutschland: Mehr als eine Mode?, in: Die Betriebswirtschaft, 66. Jg., S. 521-541. 
Tajfel, Henri (1981), Human Groups and Social Categories, 1. Aufl., Cambridge.

Tajfel, Henri/Turner, John C. (1986), The Social Identity Theory of Intergroup Behavior, in: Austin, William G./ Worchel, Stephen (Hrsg.), Psychology of Intergroup Relations, Chicago, S. 7-24.

Terjesen, Siri/Sealy, Ruth/Singh, Val (2009), Women Directors on Corporate Boards: A Review and Research Agenda, in: Corporate Governance: An International Review, Vol. 17, S. 320-337.

Thatcher, Sherry M. B./Patel, Pankaj C. (2011), Demographic Faultlines: A Meta-Analysis of the Literature, in: Journal of Applied Psychology, Vol. 96, S. 1119-1139.

Thatcher, Sherry M. B./Patel, Pankaj C. (2012), Group Faultlines: A Review, Integration, and Guide to Future Research, in: Journal of Management, Vol. 38, S. 969-1009.

Tindale, R. Scott/Talbot, Marybeth/Martinez, Rachael (2013), Decision Making, in: Levine, John M. (Hrsg.), Group Processes, New York, S. 165-192.

*Triana, Maria d. C./Miller, Toyah L./Trzebiatowski, Tiffany M. (2014), The Double-Edged Nature of Board Gender Diversity: Diversity, Firm Performance, and the Power of Women Directors as Predictors of Strategic Change, in: Organization Science, Vol. 25, S. 1-24.

Turner, John C./Hogg, Michael A./Oakes, Penelope J./Reicher, Stephen D./Wetherell, Margaret S. (1987), Rediscovering the Social Group: A Self-Categorization Theory, 1. Aufl., Oxford.

Turner, John C./Oakes, Penelope J./Haslam, Alexander S./McGarty, Craig (1994), Self and Collective: Cognition and Social Context, in: Personality and Social Psychology Bulletin, Vol. 20, S. 454-463.

*Ujunwa, Austin/Okoyeuzu, Chinwe/Nwakoby, Ifeoma (2012), Corporate Board Diversity and Firm Performance: Evidence from Nigeria, in: Review of International Comparative Management, Vol. 13, S. 605-620.

United States Census (2010), Data Access Tools, http://www.census.gov/main/www/access.html (26.03.2014).

United States Department of Labor (1987), SIC manual, https://www.osha.gov/pls/imis/sic_manual.html (03.04.2014).

Van der Vegt, Gerben S./Bunderson, J. Stuart (2005), Learning and Performance in Multidisciplinary Teams: The Importance of Collective Team Identification, in: Academy of Management Journal, Vol. 48, S. 532-547.

Van der Vegt, Gerben S./Van de Vliert, Evert/Huang, X. (2005), Location-level links between diversity and innovative climate depend on national power distance, in: Academy of Management Journal, Vol. 48, S. 1171-1182.

Van Dick, RolfiVan Knippenberg, Daan/Hägele, Silvia/Guillaume, Yves R. F/Brodbeck, Felix C. (2008), Group Diversity and Group Identification: The Moderating Role of Diversity Beliefs, in: Human Relations, Vol. 61, S. 1463-1492.

Van Dijk, Hans/Van Engen, Marloes L./Van Knippenberg, Daan (2012), Defying Conventional Wisdom: A MetaAnalytical Examination of the Differences Between Demographic and Job-Related Diversity Relationships with Performance, in: Organizational Behavior and Human Decision Processes, Vol. 119, S. 38-53.

*Van Knippenberg, Daan/Dawson, Jeremy F/West, Michael A./Homan, Astrid C. (2010), Diversity Faultlines, Shared Objectives, and Top Management Team Performance, in: Human Relations, Vol. 64, S. 307-336.

Van Knippenberg, Daan/De Dreu, Carsten K. W./Homan, Astrid C. (2004), Work Group Diversity and Group Performance: An Integrative Model and Research Agenda, in: Journal of Applied Psychology, Vol. 89, S. 1008-1022.

Van Knippenberg, Daan/Haslam, Alexander S. (2003), Realizing the Diversity Dividend - Exploring the Subtle Interplay Between Identity, Ideology, and Reality, in: Haslam, Alexander S./Van Knippenberg, Daan/Platow, Michael J./Ellemers, Naomi (Hrsg.), Social Identity at Work: Developing Theory for Organizational Practice, New York.

Van Knippenberg, Daan/Haslam, Alexander S./Platow, Michael J. (2007), Unity Through Diversity: Value-in-Diversity Beliefs, Work Group Diversity, and Group Identification, in: Group Dynamics: Theory, Research, and Practice, Vol. 11, S. 207-222. 
Van Knippenberg, Daan/Schippers, Michaéla C. (2007), Work Group Diversity, in: Annual Review of Psychology, Vol. 58, S. 515-541.

*Van Overveld, Mees N. J. (2012), Board Diversity and Financial Performance in Dutch Listed Firms, Unveröffentlichte Masterarbeit, University of Twente, Twente.

Viechtbauer, Wolfgang (2007), Accounting for Heterogeneity via Random-Effects Models and Moderator Analyses in Meta-Analysis, in: Zeitschrift für Psychologie / Journal of Psychology, 215. Jg., S. 104-121.

Webber, Sheila S./Donahue, Lisa M. (2001), Impact of Highly and Less Job-Related Diversity on Work Group Cohesion and Performance: A Meta-Analysis, in: Journal of Management, Vol. 27, S. 141.

Wegge, Jürgen/Roth, Carla/Neubach, Barbara/Schmidt, Klaus-Helmut/Kanfer, Ruth (2008), Age and Gender Diversity as Determinants of Performance and Health in a Public Organization: The Role of Task Complexity and Group Size, in: Journal of Applied Psychology, Vol. 93, S. 1301-1313.

*Welbourne, Theresa M./Cycyota, Cynthia S./Ferrante, Claudia J. (2007), Wall Street Reaction to Women in IPOs: An Examination of Gender Diversity in Top Management Teams, in: Group \& Organization Management, Vol. 32, S. 524-547.

West, Michael A./Anderson, Neil R. (1996), Innovation in Top Management Teams, in: Journal of Applied Psychology, Vol. 81, S. 680.

Whitener, Ellen M. (1990), Confusion of Confidence Intervals and Credibility Intervals in Meta-Analysis, in: Journal of Applied Psychology, Vol. 75, S. 315-321.

Williams, Katherine Y./O'Reilly, Charles A. (1998), Demography and Diversity in Organizations: A Review of 40 Years of Research, in: Staw, Barry M./Cummings, Larry L. (Hrsg.), Research in Organizational Behavior, Greenwich, S. 77-140.

Wood, Wendy (1987), Meta-Analytic Review of Sex Differences in Group Performance, in: Psychological Bulletin, Vol. 102, S. 53-71.

*Yasser, Qaiser R. (2012), Affects of Female Directors on Firms Performance in Pakistan, in: Modern Economy, Vol. 3, S. $817-825$.

\section{Summary}

Both theoretical and empirical analyses of the association between gender diversity in leadership teams and organizational performance show inconsistent results. Therefore, this study provides a comprehensive meta-analysis on the question how and depending on which contingency factors gender diversity in leadership teams is related to organizational performance. Based on 38 studies, no significant association can be identified between gender diversity and overall organizational performance. In addition, none of the investigated moderators (i.e., team size, industry setting, cultural context, and performance measure) turns out to be significant. 